Review

\title{
Differential Circuit Mechanisms of Young and Aged Visual Cortex in the Mammalian Brain
}

\author{
Chand Parvez Danka Mohammed (D)
}

check for updates

Citation: Danka Mohammed, C.P. Differential Circuit Mechanisms of Young and Aged Visual Cortex in the Mammalian Brain. NeuroSci 2021, 2, 1-26. https://doi.org/10.3390/ neurosci2010001

Received: 23 September 2020 Accepted: 15 December 2020 Published: 4 January 2021

Publisher's Note: MDPI stays neutral with regard to jurisdictional clai$\mathrm{ms}$ in published maps and institutional affiliations.

Copyright: (C) 2021 by the author. Licensee MDPI, Basel, Switzerland. This article is an open access article distributed under the terms and conditions of the Creative Commons Attribution (CC BY) license (https:// creativecommons.org/licenses/by/ $4.0 /)$.
College of Arts and Sciences, American University of Sharjah, Sharjah P.O. Box 26666, UAE; chandparvez@postech.ac.kr

\begin{abstract}
The main goal of this review is to summarize and discuss (1) age-dependent structural reorganization of mammalian visual cortical circuits underlying complex visual behavior functions in primary visual cortex (V1) and multiple extrastriate visual areas, and (2) current evidence supporting the notion of compensatory mechanisms in aged visual circuits as well as the use of rehabilitative therapy for the recovery of neural plasticity in normal and diseased aging visual circuit mechanisms in different species. It is well known that aging significantly modulates both the structural and physiological properties of visual cortical neurons in V1 and other visual cortical areas in various species. Compensatory aged neural mechanisms correlate with the complexity of visual functions; however, they do not always result in major circuit alterations resulting in age-dependent decline in performance of a visual task or neurodegenerative disorders. Computational load and neural processing gradually increase with age, and the complexity of compensatory mechanisms correlates with the intricacy of higher form visual perceptions that are more evident in higher-order visual areas. It is particularly interesting to note that the visual perceptual processing of certain visual behavior functions does not change with age. This review aims to comprehensively discuss the effect of normal aging on neuroanatomical alterations that underlie critical visual functions and more importantly to highlight differences between compensatory mechanisms in aged neural circuits and neural processes related to visual disorders. This type of approach will further enhance our understanding of inter-areal and cortico-cortical connectivity of visual circuits in normal aging and identify major circuit alterations that occur in different visual deficits, thus facilitating the design and evaluation of potential rehabilitation therapies as well as the assessment of the extent of their rejuvenation.
\end{abstract}

Keywords: aging; brain; circuit; visual; perception; monkey; human

\section{Introduction}

It is well established that aging negatively alters different cognitive mechanisms, mainly those related to visual perceptual decisions. Age-dependent decline in the performance of a wide variety of visual tasks is observed in humans [1-5], primates [6-13], carnivores [14-16], and rodents [16-19]. The majority of this decline in different visual abilities may possibly result from the functional degradation of underlying neural circuitry in central visual areas. In humans, visual abilities such as acuity [20,21], binocular sensitivity [22-24], perception of symmetry [25,26], contrast sensitivity [27,28], orientation discrimination [2,29], and motion perception [30,31] significantly decline with age. The extent of these visual deficits will depend on the internal complexity of neural circuitry that is involved in the processing of a given visual task.

Functional behavioral tasks such as perception of sensory input and controlled motor output regulate the lifelong process of learning and memory function via the systematic interconnectivity of neurons between the visual cortex and other cortical regions of the brain. Understanding the consequences of normal aging on visual perceptual abilities and working memory capacity would further elucidate key underlying mechanisms of 
an aging visual system: (1) Are all perceptual abilities deteriorated by aging? (2) Do all visual capacities decline at the same rate with age? (3) Why might the performance of aged visual circuits vary in different visual contexts? (4) How does a particular anatomical and physiological change contribute to plasticity changes related to a specific visual deficit in healthy elderly individuals? (5) Why are certain perceptual abilities the least or not impacted by aging?

Although previous studies have demonstrated a wide variety of visual impairments associated with normal aging, it would be more interesting to understand the circuit mechanism and perceptual processing of specific functional behavior tasks where neural circuitry shows no change with age. This notion suggests that aging may not always have a negative impact on visual perception nor is it entirely responsible for age-related visual deficits. Previous studies involving the assessment of cortical activation in young and aged individuals for a variety of spatial memory tasks have shown no change in psychophysical performance and spatial frequency with age [32-35]. Furthermore, it was shown that the neural substrates involved in performing behavior tasks significantly differed between young and aged groups, as behavioral performance remained identical for both the groups [35]. It appears that this reorganization of neural circuitry in an aged brain may be the consequence of recruiting additional neural networks necessary to successfully perform a given task, which may have become a compensatory mechanism for the areas that have weakened over time. This proposition is in agreement with previous reports $[34,35]$ stating that aged neural circuits do not show any significant loss in performing lowlevel memory tasks involving less complex processing of visual information, and visual processing deficits will only become evident when the complexity of visual information and computational load reaches a certain level, despite the task being cognitively simple. It is reasonable to assume that these additional neural circuits required to accomplish given tasks in an aged brain can be limited, and low-level memory tasks may probably not saturate these alternatives. On the other hand, compensatory neural mechanisms are required in an aged brain if a given task is increasingly complex, where saturation limits can be reached quickly. Previous studies in motion and texture defined tasks have demonstrated that age-dependent perceptual loss was observed more clearly in second-order tasks related to motion and texture form in comparison to lower-order tasks, suggesting that higherorder tasks require multiple brain regions and extensive processing of information [36-38].

Previous studies have shown that the complexity of neural processing increases with age, and the reduced ability of aged neural circuitry in V1 and other visual areas could be attributed to factors such as decreased spine and synapse density, leading to the regression of axons and dendrites, which in turn contributes to the differential processing of higherorder and lower visual pathways [10,11]. Age-dependent neuroanatomical, biochemical, and functional changes accumulated over time have directly or indirectly influenced the differential regulation of visual processing in V1 and multiple extrastriate visual areas [17,39]. It appears that these experiences facilitate in the adaptation of compensatory neural mechanisms in aged visual circuits, which may be necessary for the continuous upgrade of visual behavior functions essential for survival. Compensatory aged neural mechanisms correlate with the complexity of the visual task and do not always result in major circuit alterations or neurodegenerative disorders. Previous studies in young and aged monkeys have shown that excitatory postsynaptic transmission in layer $2 / 3$ pyramidal cells is similar; however, a significant increase in inhibitory synaptic currents was observed along aging [12]. Prior reports discussing age-related alterations in neuronal response properties have shown the center-surround mechanism as a reflection of complexity of higher-order neural circuits [40]. Visual experience significantly influences the organization and processing of neural pathways. The recovery of neural plasticity and the extent of rejuvenation in age-related compensatory mechanisms and diseased aging is particularly important in determining the type of rehabilitation therapy best suited for a specific visual disorder [41,42]. Although previous reviews have mainly focused on various negative effects of aging on visual processing, the present review aims to comprehensively discuss 
the effect of normal aging on the structural reorganization of neural circuits underlying different visual functions and more importantly discuss the differences between compensatory mechanisms in aged neural circuit and neural processes related to visual disorders. This paper aims to summarize and discuss age-related differences in circuit mechanisms of visual cortex under the following topics: (1) age-dependent reorganization of lower order and higher-order visual circuits, (2) dynamic balance of neuronal response properties in young and aged visual circuits, (3) circuit mechanisms for normal and diseased aging of visual functions, and (4) the rehabilitation and rejuvenation of visual circuits.

\section{Age-Dependent Reorganization of Lower-Order and Higher-Order Visual Circuits}

There is an overall decline of physiological mechanisms underlying visual functions along the process of aging. Several factors contribute to alterations in the intrinsic activity and physiological properties of neurons that significantly modulate the mechanism of visual circuitry with age. Previous studies have demonstrated the effects of normal aging on the structural and physiological properties of primary visual cortex (V1) neurons in the macaque monkey $[10,11]$. Several structural alterations including branching complexity of dendrites and total spine density were significantly reduced in neurons of aged monkeys compared to those of young ones, causing an age-related decrease in the density of synapses [10]. Despite these major structural rearrangements, there was no change observed in the action potential and synaptic current properties of excitatory and inhibitory neurons of young and aged monkeys [10]. Electrophysiological studies in monkeys have further revealed significant changes in the response properties and firing rates of layer 3 pyramidal neurons with age, whereas layer 5 pyramidal neurons relatively showed no change [11]. This is particularly important as layer 3 neurons are thought to play a more important role in the cognitive mechanisms related to visual perceptual abilities than layer 5 neurons. Prior reports in monkeys have demonstrated the effects of aging on the structure and function of area 46, which has major connections with the visual cortical association system [11,43]. It was shown that age-related structural changes in the myelin sheath effectively reduces the timing and conduction velocity of neural circuits in area 46 of monkeys that is involved in the regulation of visual, somatosensory, and visuomotor functions [11]. These findings show that there is a major reorganization of structural properties of V1 neurons with age, but these structural alterations in aged neural circuits of primates do not contribute to cognitive decline [10,11]. It appears that age-associated impairments in visual functions are not likely to arise due to any one specific structural alteration; rather, it is a combined effect of various age-related structural changes that emerge over a period of time. The combined effect of various structural changes such as demyelination, loss of spine density, and reduced synapse density followed by dendritic and axonal regression can significantly reduce the processing ability of aged neural circuits.

It is important to note that the effect of aging is not uniform across different brain regions even within the same species. Even though the physiological properties of neurons do not change significantly between young and aged visual circuits, age-related structural changes in other brain regions appears to be more profound than in V1 [10]. There is an overall loss of dendritic spines with age in monkeys, and this decrease in the total dendritic spine density in V1 was recorded to be around $25 \%$, which is significantly smaller than the $47 \%$ decrease reported for aged neurons in the pre-frontal cortex region of primates, which is commonly known to have higher spine numbers and density than V1 neurons [44]. Furthermore, in pre-frontal cortex neurons, spontaneous activity of excitatory synaptic events is substantially reduced in frequency, whereas the frequency of these events remains unchanged in V1 neurons [10]. Although there is a significant decrease in dendritic length and the number of intersections of aged V1 neurons, the total dendritic length of V1 neurons does not change significantly between young and aged monkeys [10]. It is interesting to note that neurons in the pre-frontal cortex of aged monkeys appear to be more vulnerable than aged V1 neurons, especially in regard to physiological changes. This variability in the effects of aging on neurons in these two regions of the brain is 
consistent both during development [45] and adulthood [46]. These data show that both brain regions undergo age-associated structural changes but to a different degree [10]. It seems that V1 and multiple extrastriate visual areas could be relatively less vulnerable to age-related structural and physiological changes than other brain regions such as the pre-frontal cortex, which is more influenced by age-dependent alterations. Additionally, it also appears that the moderate loss of spines and synapses in V1 neurons during aging may be necessary to maintain the functionality of aged circuits. It is possible that aged visual circuits might have facilitated in the evolution and enhancement of various neural mechanisms via lifelong accumulation of experiences, and this facilitation might possibly aid in the preservation of different physiological properties of aged neurons in the visual cortex. Preserved physiological properties of aged neurons in V1 of a monkey include increased input resistance [10], enhanced firing rates [10], reduced synaptic excitation, and enhanced synaptic inhibition [12].

Aging impairs a wide variety of visual tasks in humans [47,48]. Figure 1 depicts major events and processes involved in the reorganization of visual circuitry during healthy aging. Age-associated neuroanatomical changes are more vulnerable toward accommodating complex visual tasks in higher-order visual areas. This may further correlate with the emergence of compensatory neural mechanisms via functional recruitment and may result in a decreased performance of visual behavior functions. Visual cognitive enhancement procedures adapted to improve the efficiency of aged visual pathways play a critical role in evaluating the extent of rejuvenation in aged compensation mechanisms. Aged visual circuitry changes in several aspects of neuronal properties such as changes in neuron numbers and sizes [49], loss of retinal cells [50,51], abnormal dendritic growth [52], decrease in spine density of striate cortex cells [8], change in release-response action of neurotransmitters [53], and parvocellular pathway damage affecting spatial contrast sensitivity [54]. Although previous reports have described how some aspects of aged visual circuits are associated with the deterioration of the eye [50,51], alterations in optical quality alone cannot independently account for age-related performance deficits. Therefore, impaired visual performance in aged individuals must be partly due to changes in the structural response properties of neurons in the visual cortex [1]. In agreement with this notion, similar findings were reported in primates [6], carnivores [14], and rodents [55], where the V1 neurons of aged animals were found to be less selective to orientation pattern and direction preference than that of neurons in young animals. In humans, an age-related loss of photoreceptors followed by alterations in the connectivity of these receptor cells could result in reduced visual acuity [50,51]. Contrast sensitivity decreases linearly along aging, and visual impairment primarily occurs in the V1 region of aged individuals, where low levels of temporal frequency and amplitude were recorded at old age [3]. This might be due to the fact that lower order visual circuits subserving basic visual functions are primarily established and maintained in lower-order visual areas [56].

On the other hand, several studies have demonstrated that higher-form perceptions are more influenced by aged visual circuits resulting in decreased neural activity, revealing the relationship between functional behaviors and diminished neural functions $[3,57]$. Previous studies in humans have shown a greater impact of aging on extrastriate visual areas, where age-associated changes in grating detection were much larger for second order than that of first-order patterns [57]. Prior reports discussing the effects of aging on orientation discrimination in humans have consistently shown higher thresholds of orientation discrimination in aged individuals compared to that of younger subjects [2]. Age-dependent differences in orientation discrimination threshold was similar in both the age groups when external noise was at high levels, and input stimuli was embedded in external noise at low levels [2]. These findings are in agreement with previous reports claiming age-related differences in orientation discrimination reflecting input noise differences. It seems that increased input noise in aged individuals is associated with changes in the response properties of neurons in V1 and other visual areas. 


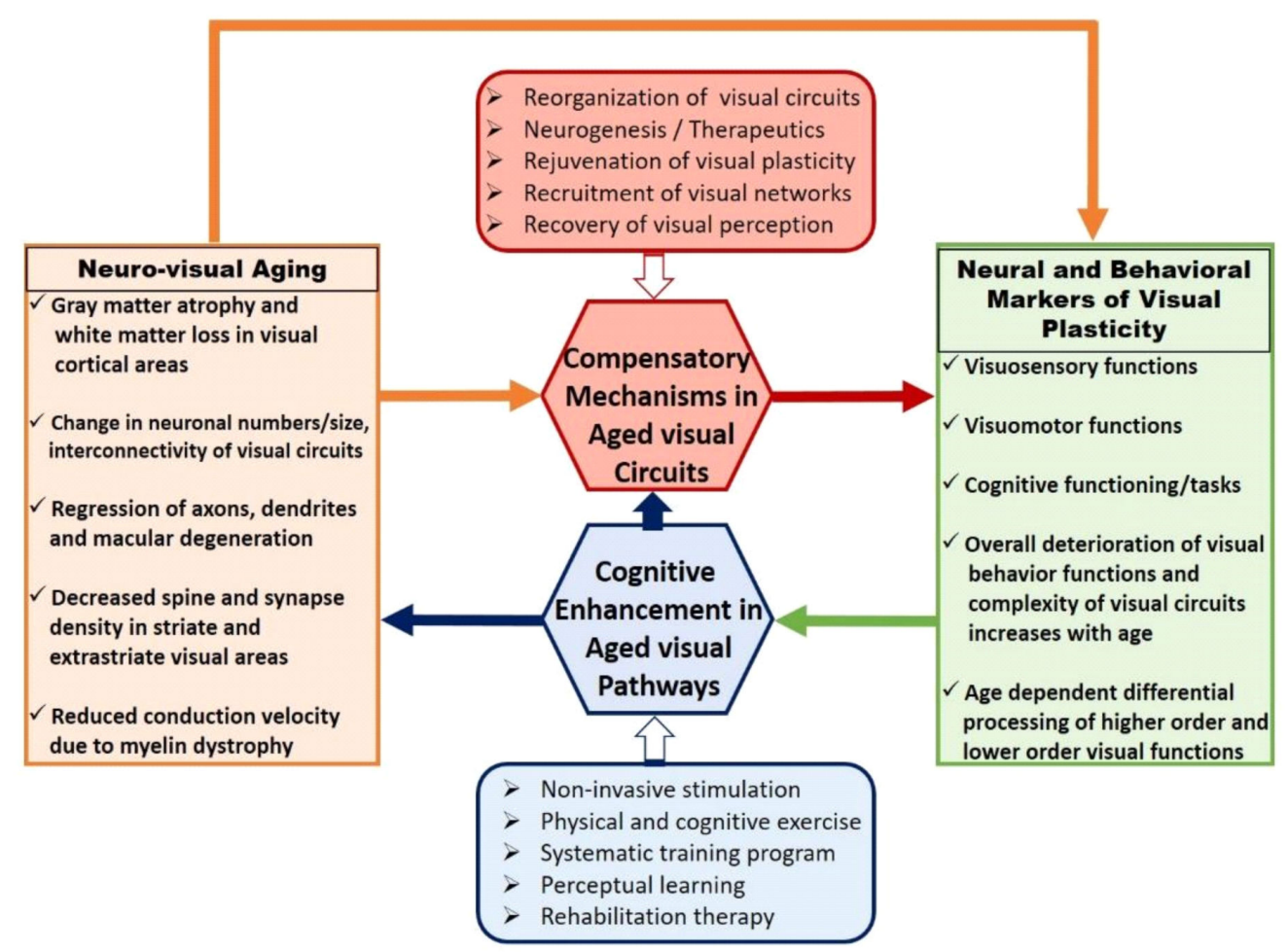

Figure 1. Schematic model depicting major alterations in visual circuit mechanisms during aging. A schematic diagram showing the reorganization and interconnectivity of visual circuit mechanisms involved in the process of normal aging. Healthy aging of the visual system ensures a pattern of neuroanatomical changes in visual circuitry underlying visual behavior alterations involving sensory, motor, and cognitive functions. Age-dependent alterations in visual cortical areas include changes in neuronal size and numbers; reduced spine and synapse density; regression of axon and dendrites; myelin dystrophy; and macular degeneration. Complexity of the visual task gives rise to compensatory neural mechanisms and alters visual behavior functions. Neurogenesis, recruitment of additional neural networks, rejuvenation therapy, and plasticity recovery methods can influence compensation mechanisms in aged neural circuitry that directly correlate with the complexity of visual task in striate and extrastriate visual areas. Visual cognitive enrichment in aged visual pathways is enhanced by different procedures such as non-invasive stimulation, perceptual learning, systematic cognitive training, physical exercise, and rehabilitation therapy.

Previous reports examining the consequences of aging on visual perception have shown some visual abilities to be more adversely affected than others. Prior studies investigating various aspects of motion perception along aging have shown that a variety of visual deficits are not accompanied by a significant decrease in contrast sensitivity, but rather, these deficits are associated with the deterioration of specific brain regions such as the medial temporal area, which is responsible for global motion perception [31,58-60]. Age-associated changes to neural circuitry do not always result in the deterioration of visual functions. Motion perception studies in aged individuals have shown the perception of large, high-contrast stimuli to be significantly better at old age when compared to young adults [1]. One of the reasons for this increase in the performance of motion perception in aged visual circuits would be the age-related decrease in the neural activity of inhibitory neurons [61], which could have deteriorating effects on a wide range of functions such as visual perception, cognition, and functional behavior, and it could possibly weaken the center-surround antagonism [62]. The lower performance level of elderly individuals has been reported in mid-level tasks such as bilateral symmetry detection [26] and also in a variety of higher-order tasks such as motion and texture forms, where second-order processing is required in comparison with other tasks that require only first-order processing [38]. These findings suggest that age-related deficits in perceptual processing become increasingly evident at a stage when the computational load 
of a given task reaches a certain level of complexity. This requires the recruitment of larger complex networks, which are currently not available in the aged brain, in order to share the processing load [35]. Aged neural circuits can be considered as the powerhouse of experience that may have adapted to certain compensatory mechanisms over time, which could result in a longer turnaround time for the execution of a particular visual function. However, these adapted mechanisms of aged circuits may be necessary for the continuous upgradation of different visual mechanisms within and across species.

Contour integration is a higher-order complex visual task that requires the segregation of a chain of Gabor patches from a noisy background where the stimuli has no global cue for orientation, color, and texture [63]. Prior studies have used higher-form perceptions such as contour integration to understand how the complexity of a higher-order task could affect visual performance in the elderly. The emergence of a global pattern is due to the interaction of local mechanisms that can be influenced by different variables such as collinearity, relative distance, and the orientation of nearby cues [63-65]. The distance between Gabor patches is particularly important as it allows integration to occur for a given input stimulus, which could be a crucial parameter in relating neural connectivity between simple cortical units [3,64]. Previous studies have documented anatomical [66], physiological [67], and imaging [68] evidence as early as in V1 that shows that horizontal connections have the ability to connect cells with receptive fields (RF) that are not overlapping and link cells that have similar orientation preferences. The segregation ability of contour integration is a second-order task that involves combining local elements in a global percept. This ability undergoes prolonged development during childhood and balances the neural activity load at old age by recruiting additional complex networks and could possibly result in age-related deficits $[3,63,64]$. Previous study discussing the functional degradation of extrastriate visual areas in aged monkeys have also correlated a decline in higher-order visual functions, such as contour integration to alterations in extrastriate V2 cells [57,60]. In order to differentiate the pattern of normal brain aging from the deterioration of a specific ability as seen in neurological impairments, it is important to understand how contour integration naturally evolves along the process of aging [22]. There is also a possibility that these adapted compensatory mechanisms of aged circuits accounting for a delay in the execution of a specific visual task might have progressively contributed toward the differential processing of visual circuits throughout the evolutionary process.

Visual surround suppression is another higher-order complex visual task that is altered by aging. Previous reports on perceptual surround suppression during normal aging have reached opposing conclusions as to whether surround suppression is reduced or increased in aged visual circuits. Visual perceptual consequences of center-surround suppression in healthy aging human visual system showed that the presence of high contrast surround suppression generates a larger perceived contrast reduction altered by surround modulation [69]. In contrast, aged visual circuits have also been reported to reduce surround suppression, where aged individuals exhibit low-duration thresholds for large high-contrast moving stimuli in comparison to younger adults [1]. One possible reason for this could be that these experiments used stimuli of different spatial and temporal properties. Aged circuits using lower spatial frequency drifting gratings showed a decrease in surround suppression [1], whereas applying static texture exhibited the presence of high contrast surround suppression in aged individuals [69]. This was further investigated by [4], where using a drifting stimulus version of the contrast perception task showed that aged individuals display greater amounts of contrast surround suppression in comparison to younger adults. Furthermore, it was demonstrated that both older individuals and young adults performed similarly using a motion discrimination task [4]. Lifelong accumulated experiences of aged circuits might have facilitated in the ongoing maintenance of various neural mechanisms, which in turn may have resulted in the continuous enhancement of different behavioral functions critical for survival.

It is thought that motion direction task being a higher form perception would partly alter neural processing in higher-order area V5, and basic visual functions such as contrast 
perception would change the neural connectivity in lower order area V1 [70,71]. It must be noted that cells from both these visual areas V1 and V5 are known to be involved in the center-surround interaction of RFs having properties of suppression [4]. Previous studies in primates have shown that V1 neurons are broadly tuned to direction selectivity and orientation preference in old animals [6], but it can be reversed to a tighter tuning by activating gamma aminobutyric acid (GABA) receptors, possibly suggesting decreased cortical inhibition in the aging brain [61]. Response variability of neurons in both lower order area V1 and higher-order middle temporal visual area (MT) were shown to be equally affected by aging in monkeys [9]. Earlier reports using psychophysical studies have also shown the possibility of feedback connections giving rise to perceptual surround suppression [72]. Binocular suppression is weak in comparison to monocular suppression, which is much stronger but has a shorter period of stimulus duration [73]. It was further suggested that monocular suppression could represent a feedforward input, as it is too fast to be involved in horizontal connections [73]. It has been suggested from earlier reports that several neural circuit mechanisms during normal aging may be similar to that in schizophrenia [74]. However, increased surround suppression with aging [69] was observed in contrast to decreased surround suppression found in schizophrenic patients [40] using the same task of motion discrimination. This suggests that aged visual circuits may not necessarily end up in neurological impairments, but they may have been considerably slowed down due to the compensatory mechanisms that may be necessary to overcome the accumulated changes over time. It also appears that age-related visual perceptual changes to the center-surround mechanism reflects the complexity of higher-order circuits beyond regular features that are routinely measured such as the timing and synchronization of neural responses, inhibition strength, and neural noise levels disrupting networked responses.

The temporal processing speed, which can alter the visual perception of moving objects, deteriorates during aging [18]. In rodents, the ability to accurately perceive the speed of a moving object deteriorates with age $[18,75]$. This functional decline could probably arise due to an age-associated decrease in the ability to detect temporal stimuli [75]. Previous studies investigating the neural basis of temporal processing speed in areas 17 and 18 of the rat visual cortex have shown a decrease in the mean speed of a moving bar of light in aged rats compared to that of young rats [18]. Furthermore, unlike cells from aged rats, neurons from young rats were able to proceed to a higher frequency stimulus [18]. Additionally, no age-related differences were observed between the cortical regions, suggesting that the difference in temporal processing speed during aging is at the visual cortical level. Unlike in primates [10], the V1 region in rodents appears to be more vulnerable to the effects of aging than other brain regions. This result also suggests the varying effects of aging within and across different brain regions in rodents. However, there are neural mechanisms in rodents that do not change significantly with age. No substantial loss of neurons or retinal ganglion cell density was recorded in the lateral geniculate nucleus (LGN) of aged rats [76], making the retino-geniculate pathway partly unaffected by the aging process. Therefore, this notion suggests that age-associated alterations in the temporal processing speed may occur higher up the visual pathway. It also appears that higher-order areas in the rat visual cortex are involved in the processing of complex functions that are more vulnerable to the effects of aging, as seen in other species.

Previous reports in humans, mice, and rats have shown that compensatory mechanisms involving dendritic proliferation were more evident in aged neural circuits [18]. In rats and humans, myelin dystrophy accounts for age-related decrease in conduction velocities $[77,78]$. Reduction in conduction velocity could lead to the slowing down of processing speed and may therefore induce a change in the preference of speed during aging, from faster in younger animals to slower in older animals [79,80]. A prior study of cats showed that the function of V1 neurons in aged cats was significantly lower than young cats [14]. However, the total density of neurons in each cortical layer of V1 showed no significant difference between young and aged cats [15]. Furthermore, the density and ratio of GABAergic neurons to total neurons was found to be significantly decreased in 
aged cats [15]. These results suggest that reduced GABAergic inhibition in V1 of aged cats could be directly associated with functional deterioration of neurons in the visual cortex.

\section{Dynamic Balance of Neuronal Response Properties in Young and Aged Visual Circuits}

Aging is often associated with reduced neuronal responses to visual stimulation resulting in a decrease in various aspects of visual functions in humans [3,5], monkeys [9,13], cats $[15,16]$, and rats $[18,19]$. During normal aging, neurons in the primary visual cortex (V1) and multiple extrastriate visual areas have been demonstrated to exhibit significantly reduced responses to visual stimulation $[38,81,82]$. Understanding the extent of age-related changes that occur in V1 and multiple extrastriate visual areas is not only important in characterizing key differences observed between healthy and diseased aging but to further elucidate underlying mechanisms that may give rise to compensatory processes in aged neural circuits. Age-associated changes are more evident in the perception of higher-order tasks than lower-order stimuli, and higher-order stimuli such as secondorder contrast defined patterns are detectable much earlier than first-order luminancedefined stimuli [38,82]. RF properties of V1 neurons have been shown to have a selective response to both directions of motion and angular orientation [83]. Both the orientation and direction selective responses of V1 cells are considered to be involved in form and motion perception. Previous reports in aged macaque monkeys show that V1 cells exhibiting reduced orientation, and direction-selective responses are associated with a significant decline of visual cortical function [6]. This decline in the visual function of aged macaques was accompanied by an increase in spontaneous activity and visual responsiveness of V1 neurons $[6,61]$. Previous studies have demonstrated that reductions in GABA-mediated intracortical inhibition of V1 neurons could contribute to the decline of visual function in aged monkeys [61]. Different factors such as decreased production and the release of neurotransmitters as well as the degradation of neurotransmitter receptors could contribute to the decline in intracortical inhibition of V1 neurons [61]. It was further shown that V1 neurons in aged animals exhibited responses similar to that of young cells after being treated with GABA agonists, showing that normal aging results in reduced ability to produce GABA in V1 cells [61]. This is an important factor to be considered, as response properties of V1 neurons are associated with sensory, motor, and cognitive declines along the process of normal aging [61]. As GABA-mediated inhibition is widespread throughout the neocortex, it can be assumed that age-related changes similar to those seen in V1 neurons could also be observed in cells of other visual areas of aged monkeys.

There is a strong association between structural alterations such as dendritic spine changes that naturally occur in neurons with age and postsynaptic effects of neurotransmitters that alters various aspects of neuronal response properties [84]. Previous studies in monkeys discussing the age-related postsynaptic transmission response of neurons have demonstrated a substantial decrease in the frequency of spontaneous excitatory postsynaptic currents along aging [84]. Alterations in neuronal response properties that occur during normal aging do not only impact the distribution pattern of neurofilament proteins but also influence several important parameters of excitatory and inhibitory synaptic mechanisms underlying behavioral functions $[85,86]$. Prior studies have shown an age-related shift in the expression pattern of the neurofilament protein, where an increase of neurofilament protein with age could render these neurons more vulnerable to the formation of neurofibrillary tangles, leading to neurodegeneration and dementia [84]. Age-dependent changes of neuronal response properties lead to alterations in excitatory and inhibitory systems, making neurons more susceptible to impaired transmission. Furthermore, this inability of neurons to function in an efficient manner could cause severe disruptions in the neuronal signaling of corticocortical pathways. The distribution pattern and expression levels of neurotransmitter receptors are also affected along aging. Previous studies have demonstrated a significant decrease in the number of neurons expressing glutamate receptors (Glu R) and N-methyl D-aspartate receptor (NMDAR) subunits along aging [85,86]. Quantitative analysis of corticocortical connections in young and aged macaques showed 
a significant downregulation of Glu R2 and NMDA R1 with age [86]. Previous studies in primates have shown that there is an overall decrease in excitatory input to pyramidal neurons with age; however, these postsynaptic excitatory receptors are functionally intact, despite being present in low numbers [86]. Furthermore, the amplitude distribution of excitatory postsynaptic currents did not differ significantly in neurons between young and aged monkeys [12]. It appears that the structural complexity of aged visual cortex is a by-product of age-dependent adaptive changes necessary for the maintenance of normal visual function.

The fact that excitatory postsynaptic currents do not change in young and aged cells of monkeys suggests that excitatory receptors in layer $2 / 3$ pyramidal cells are not functionally altered during aging [12]. On the other hand, inhibitory synaptic transmission to layer $2 / 3$ pyramidal cells was found to increase significantly with age [12]. One plausible explanation for this would be the increase in the action potential-dependent release of GABA neurotransmitters from presynaptic interneurons. Firing patterns of visual cortical cells in aged monkeys have shown a significant increase in action potential firing rates associated with a decline in stimulus selectivity [6,61]. Furthermore, pyramidal cells in layers $2 / 3$ of aged monkey showed an increase in action potential firing rates compared to young adults $[61,84]$. Aged monkeys with very high and very low firing rates performed poorly than older monkeys with intermediate firing rates that performed significantly better in comparison to young adults [84]. This shows that the optimum firing rate shifts toward higher frequency ranges during aging, which can be considered as a compensatory response to increase the conduction failure of action potential firing caused due to myelin dystrophy in aged monkeys $[61,84]$. The degeneration of myelin sheaths results in the reduction of conduction velocity affecting the response timing in neuronal circuits, which may possibly lead to cognitive impairment. Therefore, it seems that higher firing rates in aged neural circuits may be necessary to maintain normal visual function compared to significantly lower firing rates of neurons found in young neural circuits.

Previous studies discussing the effects of aging on the variability of visually evoked responses in visual areas V1 and MT of macaques showed that spike count variability in aged macaques was significantly higher than in young adults, whereas the response to noise ratio was significantly lower in aged animals compared to young macaques [9]. Spontaneous activity is generally considered as noise and is thought to reflect the intrinsic dynamic behavior of neural circuits [6,61]. Prior studies have shown that spontaneous activity could have a significant impact on different response properties of neurons $[9,61]$. The response variability of neurons along with their firing rate serves the mechanism of tuning curves with stimuli that leads responses and plays a key role in determining the encoding properties of sensory neurons [9]. Previous studies have demonstrated that high response variability can affect stimulus encoding and may disrupt the fine discrimination of sensory neurons [9]. Increased levels of neural noise and decreased response to noise ratios may have contributed to cognitive decline during aging. V1 and MT are considered to be important visual areas that are concurrently involved in the perception of orientation and motion, and any abnormal changes in the response variability of neurons in these visual areas could significantly contribute to a decline in visual perception. Previous reports have shown an excessively increased response variability in V1 and MT neurons of aged monkeys [9]. Relatively shorter refractory periods of visual cortical neurons and a nonlinear transformation pattern existing between inputs and the firing rate of neurons may have contributed to the increase in response variability of V1 and MT neurons during aging. Variations in dendritic and axonal morphology could be significantly contributing to increased response variability along the aging process. To maintain a normal range of response variability in neurons, excitation and inhibition levels are required to be balanced in relative proportions [9]. Previous reports have demonstrated the effects of age-associated imbalance between excitation and inhibition, which could possibly lead to the degradation of inhibitory intracortical circuits in aged humans and monkeys $[1,2,6,29,61]$. Prior reports in humans have shown reduced levels of L-glutamic acid decarboxylase (GAD) with 
age, which is an important enzyme that aids in the synthesis of GABA [9]. Therefore, it appears that the degradation of GABA in V1 and MT neurons during aging may be a major contributing factor for increased response variability and decreased response to noise ratio.

Neural plasticity in the human visual cortex is greater during early developmental stages of childhood and gradually declines with age $[87,88]$. Previous studies have reported an overall decrease in GABA concentration during aging, which negatively influences the neural plasticity of visual cortex in old age [88]. A relatively slower amplitude response was observed in aged adults with increased levels of GABA-mediated visual cortex inhibition $[87,88]$. In response to rapid visual stimulation, young adults showed greater changes in visually evoked potential amplitude than aged individuals, suggesting that neural plasticity is higher at young age [88]. Aged adults do not exhibit any significant change in visually evoked potential amplitude in response to visual stimuli, as increased GABA-mediated inhibition is associated with lower amplitude response and is associated with reduced neural plasticity in V1 [88]. No significant change in visually evoked potential amplitudes in aged adults suggests a correlation between increased inhibition and reduced neural plasticity along aging. Previous reports discussing age-related neurophysiological aspects of orientation processing in animals have shown an overall decrease of inhibitory function with age and proposed that perceptual abilities in healthy aged humans might be much worse than younger adults. However, different psychophysical reports on aging have shown no changes in perceptual capabilities of orientation processing leading to stronger inhibition [89]. Even though prior reports on orientation tuning suggests that changes in neuronal response properties might result from reduced local inhibition of cortical circuits, behavioral studies in aged adults neither showed clear deficits in orientation processing nor stronger surround suppression [89]. It was also shown that lateral inhibition increases in aged adults, whereas orientation tuning widths of neurons associated to local inhibition remained globally intact with age [89]. Furthermore, there is no change in the systematic tuning width of aged adults, but instead, it showed a higher neuronal noise during aging [89]. This suggests that age-related compensatory mechanisms are more complex than previously thought, involving stronger lateral inhibition along with mixed effects on local inhibition.

Age-dependent dysfunction of different cognitive processes is related to neuronal mechanisms of visual perception. Previous studies investigating age-related changes in neuronal response properties that underlie visual decision making have shown that alterations in neural noise, cross-inhibition of different neural populations, and the adaptation of neural populations for different visual percepts shape up the process of visual decision making [90]. The rate of alternation for different percept choices significantly decreases with age, and this effect is more pronounced for intermittent stimulus durations [90]. In contrast to previous reports [91] that claimed slower dynamics for aged individuals due to increased inhibition in the visual cortex, [90] showed that age-related visual decision making is not correlated with alterations in inhibition strength but by changes in other low-level neural properties. It appears that visual perceptual decisions during aging is mainly caused by alterations in low-level neural properties such as neural noise and neural adaptations and not by the changes that occur in inhibition strength.

In order to understand the different neural mechanisms that are involved in agedependent alterations of the visual cortex, it is important to understand the organizing principle of a visual field map. Prior reports measuring cortical surface areas have shown no significant changes in V1 surface area and V1 volume during normal aging [5]. Previous studies have demonstrated a correlation between age-dependent decline in visual acuity and significant decrease in both foveal surface area and the distribution of visual field maps of aged individuals in comparison to young adults [5]. Furthermore, significant differences in population-receptive field sizes were observed across the visual field maps, which were correlated with decreased visual acuity along with aging [5]. An age-related increase in the population receptive field size appears to be a compensatory mechanism adapted by 
older visual circuits for reduced foveal surface area and the distribution of visual field maps. Previous reports discussing age-dependent deficits in spatial and temporal contrast sensitivity have correlated these changes with the broadening of population receptive field sizes [5,92]. Visual areas V1, V2, and V4 have been shown to be involved in neural mechanisms that subserve spatial attention in humans and macaques [92]. Furthermore, decreased foveal surface area and the distribution of visual field maps in aging individuals accompanied by increased population receptive field sizes could contribute to tuning deficits of spatial attention [5]. These age-related changes in the population receptive field size of V1 may be internal to V1 or can be the result of age-related changes in other visual areas such as feedback response from higher-order visual areas to V1 [5,92]. Therefore, it can be assumed that neuronal response properties such as RF size changes dynamically with age and plays a compensatory role in aged visual circuits that are vulnerable to visual deficits.

Previous studies discussing the impact of aging on spatial vision have shown that age alters various neuronal response properties such as contrast gain, perception of global shapes, center-surround interactions, and the integration of contour elements $[93,94]$. However, the ability to make discrimination and contrast-matching judgments is not altered by the aging process, and aged adults showed no significant contrast gain for perceptual tasks associated with parvocellular and magnocellular pathways [93,94]. This suggests that aged individuals can have different perceptual experiences in complex environments. Furthermore, aged adults exhibit increased threshold levels in identifying global shapes, including both glass and frequency patterns [3,94]. Aged individuals in comparison to younger adults exhibited a stronger suppressive effect of surround, showing high-contrast suppression when both the center and surround were exposed to the same eye, whereas no such effect was observed when the center and surround were exposed to separate eyes independently $[3,93,94]$. Hence, it seems that there is a generalized contextual change in surround suppression, contrast, and orientation with normal aging that does not result in a significant decline of performance in aged adults [95]. Even though visual processing is more susceptible to the effects of aging, neural changes in visual circuits related to cognitive tasks do not show a major performance decline in aged individuals as compared to young adults, despite age-related impairment in neural responses of older subjects.

Aging differentially influences various stages of visual processing and mediates much of the degradation of neural pathways. Previous studies in carnivores involving the systematic comparison of neuronal response properties such as contrast response function from lower-order to higher-order visual areas showed varying degradation of different visual areas during aging. In cats, the effect of aging was found to be negligible on LGN, while it was significant on the striate cortex (Area 17) and more severe on posteromedial lateral suprasylvian cortex (PMLS analog to MT in primates) [96]. The decreased contrast sensitivity of neurons in these visual areas was accompanied by high spontaneous activity, low signal to noise ratio, and increased visual response [96]. Previous reports have also shown that the functional deterioration of visual cortical neurons in aged cats is mainly associated with motion direction stimuli [15]. Furthermore, aged cats exhibited significantly reduced density and proportion of GABA neurons accompanied by decreased GABA inhibition [15]. Initial reports in rodents did not show any significant change in GABA with age $[15,97]$, but later studies in rats corroborating previous reports in humans showed a significant decrease in the GAD enzyme necessary for GABA synthesis $[15,98]$. However, visual neurons respond to orientation selective stimuli despite decreased GABA inhibition, suggesting that GABA inhibition is not necessary for the orientation-selective response of single neurons [15]. Furthermore, it was shown that excitatory input can generate neuronal selectivity to motion direction and orientation stimulus, whereas the inhibitory input can suppress the response variability of neurons to orientation and motion direction stimulus, thus enhancing the stimulus-tuning selectivity of neurons [15]. Alterations in GABAergic inhibition during aging may serve in strengthening postsynaptic efficiency of visual cortical neurons, thereby increasing visual responsiveness to orientation and motion 
direction stimulus, which in turn decreases the stimulus selectivity of neuronal response to motion direction and orientation [15]. It appears that there is a greater effect of aging on neurons in successively higher stages of visual areas compared to other regions of the visual cortex. Increased GABA inhibition alongside aging seems to promote a high variability of visually evoked responses of neurons accompanied by decreased signal to noise ratio. A more severe deterioration of aged neurons in higher-order visual areas could possibly provide an explanation for greater deficits of complex visual functions along aging.

\section{Circuit Mechanisms for Normal and Diseased Aging of Visual Functions}

Certain age-related visual deficits have been associated as part of normal aging process, such as alterations in the population receptive field size of V1 and V2 neurons, which aid in the representation of foveal input [99-101]. The age-dependent accumulation of these neural changes contributes to age-associated loss of lower-order visual functions such as orientation preference, visual acuity, and contrast sensitivity as well as higher-form visual perceptions such as motion processing, face recognition, and speed of reading [100]. Figure 2 is a schematic model illustrating the effect of normal aging on visual perception. Accumulated effects of healthy aging alter neuronal signaling in interareal and corticocortical visual pathways. The processing of complex visual tasks with increased computational loads in higher-order visual areas can trigger age-associated compensation mechanisms that may result in decreased visual perception and functional degradation of visual cognition. Previous studies have shown that the aging population often has deficits for orientation stimuli, motion stimuli, and contrast stimuli [102]. These deficits in the aged population lead to reduced sensitivity of all the above-mentioned types of second-order stimuli [102]. Additionally, there are acquired causes of vision loss during aging, which may include diseases such as cataract, diabetic retinopathy, glaucoma, and macular degeneration where the disease directly or indirectly affects the eye and decreases visual functioning. These changes significantly impact the transmission and processing of information to the central visual pathway and in different visual areas [100]. Generally, these eye diseases are characterized as neurodegeneration that starts from the eye and gradually spreads and progresses to the visual cortex. Therefore, it appears that alterations in vision that occur during normal aging and disease could possibly involve neurodegenerative processes in V1 in addition to other optical changes.

V1 and multiple extrastriate visual areas undergo a variety of neuroanatomical, biochemical, and functional alterations with age $[17,39]$. From an anatomical perspective, a major loss of visual cortical neurons mainly occurs in the last phase of life [44,49,52]; from a biochemical point of view, GABAergic, serotonergic, and cholinergic neurotransmissions appear to be the most affected by aging $[15,55,61]$; in terms of behavioral correlates, a substantial decline of various functional mechanisms of the visual cortex is documented in the aged population $[15,34,55]$. Altered visual processing due to age-related structural and functional changes of the visual cortex may constitute for a variety of neurological impairments. Since visual deficits are often described as one of the early symptoms of dementia, it is important to understand the effects of normal aging on visual processing compared to relative changes of visual cortex in neurodegenerative diseases such as Alzheimer's disease (AD), Huntington's disease (HD), and Parkinson's disease (PD). Previous studies have shown that neuronal death during normal aging is significantly less as compared to the high cell loss in $\mathrm{AD}$ [84]. $\mathrm{AD}$, the most common form of dementia, has been characterized as a progressive neurodegenerative disease that selectively damages neural circuits, and it is distinguished by neurite dystrophy present in about $80 \%$ of the aged population having dementia $[5,84,103,104]$. Age-dependent loss of neurons, decline in synaptic plasticity, and cognitive impairments are often observed in normal aging and neurodegenerative diseases such as AD $[84,105,106]$. In contrast, previous studies in many species have reported age-related cognitive deficits that are mainly mediated via synaptic and dendritic changes without any significant loss of neurons [15,34,55,61,84]. Morphological alterations in dendrite structure and decreased spine density could render intact neural circuitry vul- 
nerable, and these changes are exhibited in the compromised synaptic plasticity of different neural pathways $[15,55,61,84]$. However, unlike neurodegenerative diseases such as AD where neuronal loss contributes significantly to cognitive decline, age-dependent cognitive impairments progress through changes at the cellular and macro-molecular level $[105,106]$ that could potentially disrupt visual cortical connectivity.

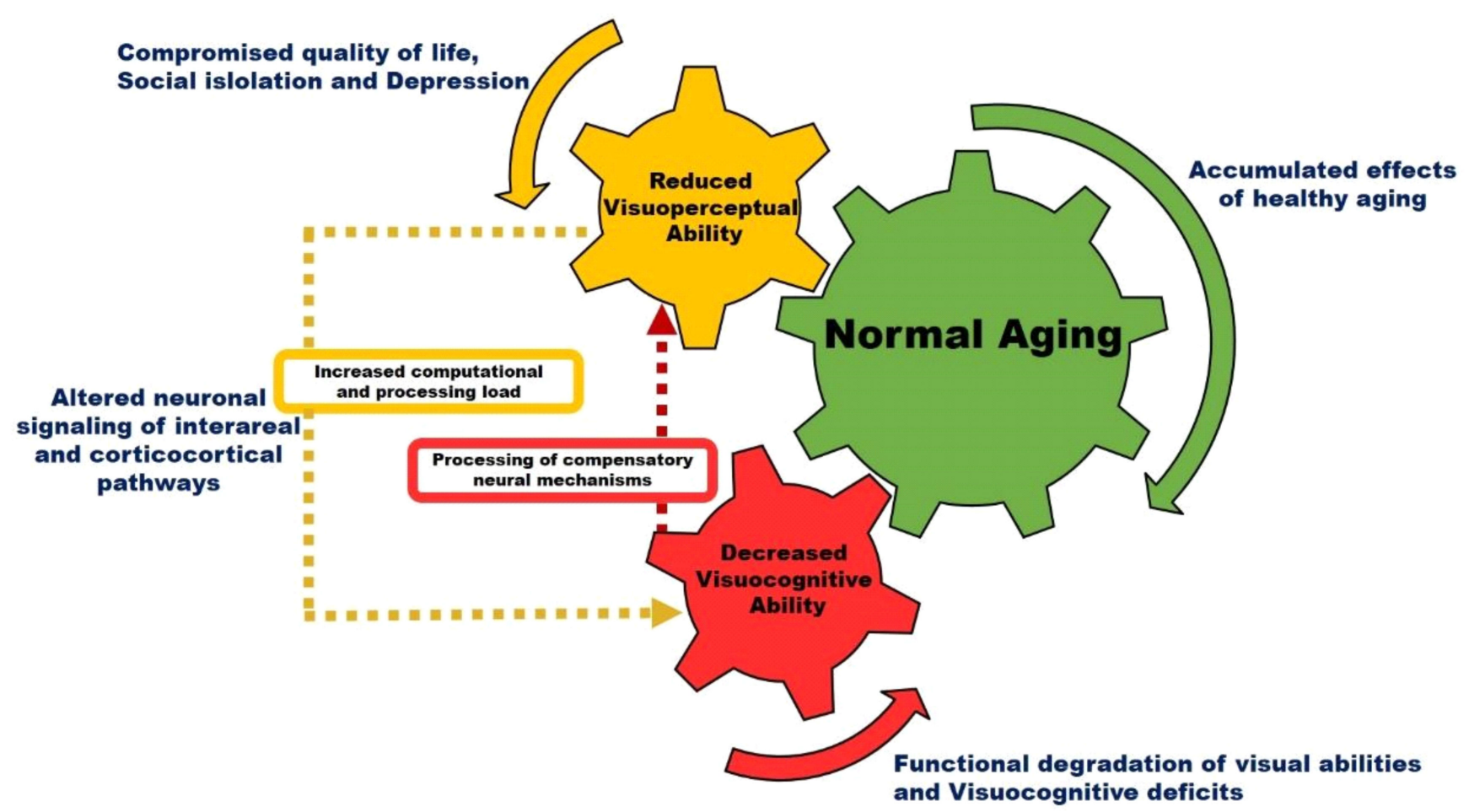

Figure 2. Schematic model illustrating age-dependent correlation between visual impairments and cognitive dysfunction. A schematic diagram illustrating potential interlinks between visual perceptual abilities and visual cognitive deficits during normal aging. Accumulated effects of general wear and tear during healthy aging can alter the neuronal signaling of visual pathways and trigger the activation of compensatory neural mechanisms via functional recruitment in response to increased computational load. Age-associated decline in visual perceptual ability results in the degradation of visual functions and possible occurrence of visual cognitive deficits. Significant alterations in higher-order and lower-order visual circuitry during normal aging can reduce the efficiency and performance of key visual pathways necessary in the processing and maintenance visual functions.

A great amount of research over the past few decades has shown the dynamic nature of neuroanatomical and functional organization of neural circuits in human, non-human primates, and other species that alter in response to various stimuli $[17,39]$. Neuroimaging studies have shown lower levels of blood oxygen level dependent (BOLD) activity in V1 of elderly subjects as compared to young adults, while no significant effect on fixation stability was observed in aged adults, which is an important parameter used for visual field map assessments [5,103]. Previous studies discussing various modeling methods of the population receptive field have demonstrated several alterations such as decreased foveal surface area and increased RF sizes in the V1 of elderly subjects as compared to young adults $[5,103]$. These changes may reflect behavioral alterations such as reduced contrast sensitivity and decreased visual acuity and anatomical changes such as reduced thickness of the retinal nerve fiber accompanied by a substantial decline in retinal photoreceptors [5]. Cortical representation of visual field maps providing functional measurements could be used to identify precise effects of neurodegeneration on V1 and other visual areas in the very early stages of disease [103]. Symptoms of visual impairments in AD can range from low-level visual deficits such as contrast sensitivity, processing speed, field coverage, and visuospatial perception to high-level deficits such as visual attention and the feature recog- 
nition of complex objects [5]. Previous reports discussing emerging patterns of evidence for neurodegeneration in $\mathrm{AD}$ visual pathways demonstrated a steady increase of plaques and neurofibrillary tangles from V1 to associative visual cortex, which is accompanied by a significant loss of retinal ganglion cells and the widespread degeneration of optic nerve axons $[5,103,104]$. It appears that this disruption of normal inputs to various visual processing streams will be reflected in terms of alterations in the anatomical organization, behavioral function, and neural connectivity of the visual cortex. Prior reports using fMRI imaging studies have shown the feasibility and characterization for assessing the organization of visual field maps and RF of neurons in AD patients, which can be further correlated to potential alterations in the visual cortex as part of the pathophysiological features of $\mathrm{AD}[5,103,104]$. This type of understanding will not only contribute to early detection of $\mathrm{AD}$ but it will also help decipher major differences between $\mathrm{AD}$ and other forms of dementia. Previous studies in aged mice have shown an increased deposition of $\mathrm{AD}$ markers such as $\mathrm{A} \beta 42$ peptide, microtubule-associated protein (TAU), and glial fibrillary acidic protein (GFAP) in the endothelial cells, blood vessels, and neurons of the visual cortex [107]. Furthermore, it was shown that aging could be possibly associated to the activation of the amyloidogenic pathway, which results in the overproduction and intracellular accumulation of $A \beta 42$ in visual cortical neurons [107]. It appears that the accumulation of $A \beta 42$ affects important visual pathways and mechanisms that further serves in the initialization of various neurodegenerative diseases such as AD and other forms of dementia.

Visual processing deficits are not only present in AD but also in its pre-dementia phase, which is known as mild cognitive impairment (MCI). Previous studies evaluating the connectivity pattern of visual processing in MCI patients compared to $\mathrm{AD}$ and healthy aging subjects showed that connectivity with ventral visual areas was significantly diminished in MCI patients, while the connections with medial parietal regions was intact [104]. Although physiological changes in the connectivity of ventral visual areas during healthy aging were observed, psychophysiological interaction effects were found to be significant only in MCI and AD patients [104]. These connectivity changes may exhibit MCI and AD compensatory mechanisms that are ineffective in disease pathology, and they could be further reduced in its compensatory effect as the changes become more evident with the progression of the disease. It appears that $\mathrm{MCI}$ acts as a transitional stage between normal aging and $\mathrm{AD}$, and connectivity changes depend on the visual task and visual areas involved. Previous reports in $\mathrm{MCI}$ and $\mathrm{AD}$ patients have also shown the reorganization of functional networks by recruiting additional visual areas related to attention deficits and the dorsal visual pathway serving as a functional brain reserve $[103,104]$. However, it seems that these compensatory mechanisms may not be effective with the progression of the disease nor sufficient to overcome the degenerative effects of pathological aging as seen in AD. Prior reports in AD have demonstrated visual field map changes and a severe degeneration of excitatory neurons compared to inhibitory interneurons in V1 that may result in color vision disorders of $\mathrm{AD}$ patients $[5,103]$. This process may also contribute to the significant decrease in RF size of neurons in higher-order visual areas involved in color vision and form processing [103]. Visual spatial attention deficits in AD may be correlated with coherence changes in V1 and V2, and the significant decrease in RF sizes in the periphery of V2, V3, and other higher-order visual areas could be contributing to the shrinkage of visual field space seen in AD patients $[5,103]$. It appears that these degenerative changes in the visual cortical system may reflect the deficits seen in the optic nerve, retina, and alterations in feedback response from higher-order visual field maps as evident in patients with dementia.

In Huntington's disease (HD), structural and functional alterations of the visual cortex and visual cognitive deficits are present in both early and advanced stages of the disease $[108,109]$. A decline in both structural and functional integrity of the visual pathways are characterized by cell loss, cortical thinning, retinal thinning, decreased visually evoked potential amplitude, and decline in second-order visual perceptual and motor abili- 
ties $[108,109]$. Neuroimaging studies have shown atrophy of the striate cortex in HD to be one of the major sources for motor deficits and thinning of the visual cortex in HD to be associated with cognitive performance of visuospatial tasks [108]. This implies an association between higher-order cognitive tasks and visual cortical degeneration in HD. Alterations in neuronal connectivity associated with increased atrophy and decreased neuronal function were observed in the associative visual cortical regions of HD patients [108]. V1 did not exhibit group differences in vascular activity and cortical thickness between early and advanced HD patients; however, significant thinning of the associative visual cortex was correlated with decreased visual perception $[108,109]$. It appears that V1 remains largely preserved both in its structural and functional form, despite the presence of atrophy in the early stages of HD.

Visual hallucinations are common and frequently observed in Parkinson disease (PD), specifically as the disease progresses to advance stages and generally ranges from basic flashes of light to more complex hallucinations that are typically exhibited by wellformed images [101]. Previously proposed models explaining the complexity of visual hallucinations have discussed the possible disruption and alteration in communication between the higher-order and lower-order visual cortical regions that are involved in visual processing [101]. Previous studies assessing the neuropathological damage of the visual system in dementia with Lewy bodies have demonstrated a significant decrease in GABA activity of the visual cortex particularly in V1 [100]. This alteration in neural circuitry could be a compensatory mechanism in response to weak visual stimuli or disrupted neural connectivity within the visual areas that have reduced GABA inhibition. Previous reports investigating visual hallucinations in PD have shown reduced concentration levels of GABA along with significant loss of gray matter in higher-order visual areas [101]. Furthermore, low levels of GABA activity were correlated with decreased visual acuity in PD patients with visual hallucinations, and a weak input signal to the visual cortex would possibly serve in the optimization of visual processing supported by a decreased inhibition of GABA [101]. No direct association between the severity of visual hallucinations and GABA activity suggests that decreased levels of GABA could possibly make individuals hallucinate; however, various other factors such as visual environment and visual attention might be critical in the emergence and regulation of visual hallucinations [101]. It appears that visual hallucinations are a side effect of neural alterations that preserves and maintains visual function, which may deteriorate with weak visual stimuli or poor neural connectivity.

Unlike PD patients with no visual hallucinations, PD patients with visual hallucinations showed no significant activation of higher-order visual areas such as V5 [101], whereas previous studies have reported that decreased activation and dysfunction in area V5 could contribute to visual hallucinations due to improper motion tracking of objects [99]. Since the ventral visual stream plays a major role in object recognition, it is likely to be involved in the genesis of visual hallucination [101]. Previous studies have shown the visual ventral stream to include projections from V1 to the temporal lobe and gray matter atrophy in PD patients with visual hallucinations, and it was mainly found to be in the temporal lobe region [99,101]. Temporal areas in ventral stream pathways consist of significantly high number of Lewy bodies with an increasing density concentrated in the anterior temporal lobe region, which is suspected to be contributing visual hallucinations in dementia with Lewy bodies [99-101]. It appears that the decline in GABA activity may be driven by the pathological state of ventral visual pathways that may alter neural connectivity between V1 and higher-order visual areas. Furthermore, it was shown that changes in GABA activity were associated with structural alterations in the visual ventral stream of PD patients having visual hallucinations $[99,101]$. Higher-order areas such as V4 projects to hippocampal regions and are found to be involved in the regulation of visual functions such as object recognition and coordination of signals between V1 and higher visual areas. Therefore, it is possible that atrophy in ventral visual areas including gray matter loss in temporal lobe regions could possibly disrupt the communication channels between 
the ventral visual pathways and lateral frontal cortex region, as they are mechanistically involved in the occurrence of visual hallucinations.

Prior studies investigating dementia with Lewy bodies in patients recurrently experiencing visual hallucinations do not show any neuropathological changes in V1, and they exhibit no significant evidence for neuronal change in dementia with Lewy bodies [100]. Recurrent visual hallucinations do not always occur in blind individuals and contribute to decreased visual input of V1 in patients having dementia with Lewy bodies, suggesting the existence of other biochemical or pathological changes that may be necessary for complex visual hallucinations to occur frequently [99-101]. Furthermore, the presence of pathological structures has indicated a correlation between temporal lobe Lewy bodies and recurrent visual hallucnations, demonstrating that increased density of Lewy bodies in the temporal lobe region is associated with frequent occurrence of visual hallucinations [100]. However, there appears to be no significant structural change to the temporal lobe in patients having dementia with Lewy bodies, which may not be necessarily similar to PD patients with recurrent visual hallucinations. Patients with dementia Lewy bodies having a severe recurrence of visual hallucinations show decreased connectivity of the temporal lobe, particularly in the ventral temporal junction following visual stimuli [100]. Therefore, it seems that there are functional changes occurring in the GABAergic neurons of patients having dementia with Lewy bodies in the absence of pathological changes in V1. This also suggests that age-related alterations in the eye could possibly decrease visual acuity by suitably modifying the visual input. These alterations could probably lead to an imbalance of the inhibitory and excitatory activity of V1 neurons, and fine-tuning the modulation of GABA activity in neurons may improve the recurrence of complex visual hallucinations in patients having dementia with Lewy bodies.

Previous studies in monkeys have shown that the intact structure of myelin sheaths that surrounds the nerve fibers in V1 is compromised during aging [7]. However, the structural alterations of myelin sheaths were not as a result of degenerating axons and myelin. Additionally, very few degenerating axons were found to be surrounded by myelin sheaths [7]. It appears that one of the major effects of aging on nerve fibers involves the degeneration of myelin sheaths. Even though previous studies have suggested very few axons to exhibit signs of degeneration in V1 nerve bundles, white matter loss was documented in both humans and monkeys during aging [7]. Previous findings in monkeys have suggested that alterations in myelin sheaths in area 17 may exhibit widespread morphological changes throughout the visual cortex that could possibly alter the information processing channels, especially for more complex and higher-order cognitive tasks. Prior studies investigating the distribution of the white matter neuron population with age do not show any significant loss of cells during aging; however, evidence of cell loss was found in the white matter of AD patients [13]. Prior reports in monkeys have consistently showed no age-associated loss of neurons in cortical gray matter, and white matter neurons could be possibly following neurons in gray matter and show no significant change with age [13]. Furthermore, a substantial increase in the number of glia cells in the infragranular layers of the visual cortex was reported during aging [13]. It appears that this increase in glia cells could be a possible reflection of glial cell response to pathology in myelinated fibers and the degeneration of myelin observed in aged monkeys that may influence the distribution pattern of white matter neurons. Regional diversity was also exhibited in the distribution of white matter neurons. The tendency of white matter distribution appeared to be broadly similar in the temporal and parietal regions but exhibited significantly lower counts in the frontal area of V1 [13]. Important factors that could possibly contribute to the regional diversity of cell numbers and age-associated vulnerability of white matter neurons include differences in white matter environment and dissimilarities in afferent or efferent connectivity. In monkeys, the correlation between the degeneration of myelin sheaths and decline in performance of cognitive tasks depends on the role of myelin in axonal conduction [7]. It appears that age-associated defects in myelin sheaths could bring about the alteration in the rate of axonal conduction. Previous studies in cats investigating 
the conduction velocity of axons have shown young cats to have twice more pyramidal neurons than aged cats that were fast conducting, and aged cats exhibited a greater decline in median conduction velocity compared to young ones [7]. Therefore, it appears that defects in myelin sheaths could possibly result in the reduction of conduction velocity in axons, which could alter the timing of critical events in neural circuits and perhaps lead to extended activity (excitation or inhibition) of neurons, which may be the underlying cause of cognitive deficits during aging.

\section{Rehabilitation and Rejuvenation of Visual Circuits}

Eye-related impairments can significantly alter various forms of visual perception by introducing major changes to the neuroanatomy of visual circuits $[41,42,65]$. These changes directly impact the anatomical structure of visual pathways leading to substantial changes in behavioral function [41,42]. For instance, macular degeneration obstructs the normal flow of information in visual pathways, contributing to retinal damage and results in visual field acuity deficits $[42,58,110]$. The visual system that is subjected to diseased aging gradually adapts to the changes caused by pathological damage and follows compensatory mechanisms, which may alter important characteristic features of neural circuitry, visual processing, and form perception $[42,65]$. Meanwhile, various alterations that occur in the neural circuitry of an aging visual system may either emerge spontaneously or depend on visual experience [65]. Early reports in V1 of young cats showed no recovery of visual function from extended deprivation following the early critical period. However, more recent studies have demonstrated evidence for neuronal plasticity occurring in adults with impaired visual abilities [65]. The rejuvenation of neuronal plasticity during normal and diseased aging is particularly important in the design and execution of rehabilitation procedures. It is important to understand the varying contribution of different neuroanatomical sites and their underlying neural processing mechanisms necessary for the recovery of visual plasticity that may either occur spontaneously or with systematic training $[41,65]$.

Both normal and abnormal visual experience significantly influences the organization and processing of visual pathways. Visual cortical circuitry exhibits more stable characteristics and relatively shows less adaptive behavior to changing sensory inputs beyond a critical period [41,42]. Reduced visual plasticity is expected after late onset of low vision from various eye disorders such as age-associated macular degeneration, cataract, and congenital blindness $[42,58,110]$. However, previous reports have only shown the partial recovery of visual plasticity in individuals with low vision, which is very important in assessing the success of rehabilitative therapies $[41,42,65]$. In general, plasticity restoration treatment would work for an individual where the visual system does not readily adapt or alter after the onset of low vision. For example, in aged patients with cataract, visual functions often return to normal after surgery; however, therapeutic interventions such as surgery, training procedures, and perceptual learning may not be beneficial in patients with low vision where the aged visual system is unable to adapt to the changes [42]. It is important to understand the adaptation response of neural circuits to altered visual experience and how this ability of visual circuitry may decline during aging when visual disorders become prevalent. Previous studies in this aspect have suggested various ways that are used to alter the visual experience of a person. Systematic training and perceptual learning is one such method that involves repeated testing of the subject with the same stimuli, and significant improvements in the visual performance of aged patients for various tasks such as face recognition, letter identification, orientation discrimination, and texture identification were observed after several weeks of intensive training [58,110-113]. Other ways of changing the visual experience include the systematic blocking of patient's access to his daily visual environment, and after several hours of continuous exposure to an orientation-deprived environment, an increase in sensitivity to deprived orientation can be observed in comparison to controls [42]. Furthermore, following the exposure, the subjects also exhibited increased contrast sensitivity and improved functional magnetic resonance imaging (fMRI) blood-oxygen-level-dependent (BOLD) responses in visual areas 
V1 and V2 [42]. It appears that these changes are correlated with an increase in response gain in the visual cortex that is directly associated with decreased contrast sensitivity in patient's natural visual environment, highlighting the increased response of human visual cortex to weak input signals.

Low vision can be defined as a type of visual deficit that is most commonly characterized in terms of residual visual field and visual acuity $[41,65]$. It emerges as a consequence of neurological impairment that results in decreased function of visual behavior and requires assistance in everyday activities $[41,65]$. Neural plasticity alterations in low vision correlate with changes in visual perception and functional behavior that occurs after the onset of visual disorder and are not directly associated with the pathological condition of the individual [65]. Previous studies have shown the incidence of low vision to increase with age and are found to be more prevalent in individuals aged 85 and above [41,65]. The evaluation of several factors such as age, visual experience, spontaneous and training-dependent plasticity changes, perceptual and physiological markers of plasticity can provide important information that can maximize the rehabilitative benefits in patients with low vision $[41,65]$. Previous studies discussing neural and behavioral markers of plasticity in individuals with low vision have demonstrated the disruption of retinotopic organization of V1 to occur in the early stages of low vision [41]. Previous studies have reported cross modal changes in visual circuits after light deprivation, where normal blindfolded subjects showed enhanced performance in various discrimination tasks with a simultaneous increase in fMRI BOLD signal in response to visual stimuli, providing further evidence for visual plasticity with age [114]. Furthermore, it was shown that the cross modal changes occurring in low vision and blind individuals are comparable, extending the sharing of these changes to extrastriate cortical regions in the visual pathway that are actively involved with behavioral tasks such as object recognition and motion processing [42]. The three major types of behavioral assessments that are used to evaluate neural plasticity changes in individuals with low vision include (i) determining plasticity differences in visual function of low vision subjects and normally sighted individuals; (ii) long-term studies evaluating visual function changes that are not directly associated with the underlying pathological condition; and (iii) a systematic training program of perceptual learning aimed at improving the specific behavioral aspects of visual function $[41,65]$. Visual behavior and functional changes are measured in terms of visual acuity, contrast sensitivity, and visual field perimetry [41,56,65].

One of the major causes of visual disorders in the aged population is cataract; however, treatment options involving the extraction of cataract and implantation of artificial lens have proven to be successful among the elderly [115]. It is interesting to note here that patients who undergo cataract removal surgery have often had cataracts for many years prior to the extraction and yet show substantial recovery in visual functions such as visual acuity and contrast sensitivity returning to normal levels $[42,115]$. Important perceptual biases linked with pre-surgery and after cataract extraction include (i) prior to surgery, individuals with cataract report warm colors in their visual environment, which change to more cooler colors following cataract extraction; (ii) visual stimuli settings for cataract subjects seem to be in the bluish region initially and then shift to the yellow region after the surgery; (iii) best focused images for cataract patients are in the blurred state, which soon changes to sharp focus following cataract surgery [42,115]. It appears that visual pathways have the ability to adjust and accommodate modified visual responses that are abnormally generated by the eye in disease, therapy, and more importantly in old age. Prior reports have demonstrated that repeated perceptual learning can improve the reading performance in individuals with macular degeneration [58]. Reading speed was shown to improve by more than $50 \%$ in individuals with early onset of macular degeneration (Stargardt disease); furthermore, the enhanced performance of reading was maintained even with smaller print size $[58,110]$. Other studies in this aspect showed that the oculomotor training task proved to be more effective for enhanced reading speed, increased visual acuity, and greater fixation stability, showing positive correlations with fMRI BOLD alterations in visual areas V1, V2, and V3 in subjects with Stargardt disease [42]. It is very encouraging 
from a rehabilitation point of view to note that plasticity recovery in aged subjects with macular degeneration restores back to normal levels of adult vision.

Visual functions develop and deteriorate at different rates [56]. These differences may directly or indirectly impact the extent of recovery and process of rejuvenation in aged, diseased, or damaged visual circuitry. The age of onset of visual disorder can have a strong influence on both the magnitude of plasticity changes and the possibility for the restoration of normal vision [42]. Prior studies in cat visual cortical cells investigating the irreversible effects of early visual deprivation showed that the recovery rate of visual function after prolonged visual deprivation was insignificant or largely incomplete [116,117]. Previous reports discussing case studies of vision restoration in humans following longer periods of blindness (congenital or early onset) showed poor results [42]. It appears that an incomplete development of experience-dependent visual function prior to visual deprivation leads to a partial or incomplete development of neural circuits after vision restoration, and visual capacities such as visual acuity that had matured earlier may also show decreased levels when subjected to prolonged periods of visual deprivation [41,42]. However, recent studies have demonstrated adult plasticity following long-term visual deprivation in amblyopic individuals with decreased levels of visual acuity and contrast sensitivity $[118,119]$. The general view is that rehabilitation therapy or other treatment options for amblyopia are ineffective following an extended critical period, whereas recent evidence exhibit that specific types of visual training can possibly yield plasticity improvements in visual functions for individuals with amblyopia and strabismus [118,119]. Furthermore, it was also shown that different forms of plasticity occurred in lower-order sensory functions and higher-form perceptions in individuals with congenital cataract and early blindness following extended periods of visual deprivation [42].

Another cause for low vision is cortical lesions that eventually lead to a loss of visual field. Previous studies discussing vision restoration therapy for improving the plasticity of neurons with specific focus on the transition zone between visible and invisible areas showed this method to be effective in the enlargement of the visual field image [42]. Recent follow-up studies have demonstrated a much greater expansion of the visual field when vision restoration therapy was combined with transcranial direct current stimulation [42]. Vision recovery studies have shown post-surgical improvements in visual acuity and contrast sensitivity following cataract removal in patients with little or no pattern vision [42]. Sight restoring surgery in both early and prolonged visual deprivation after cataract removal has demonstrated significant improvement in plasticity. A substantial 30-fold increase in peak contrast sensitivity along with improved cut-off frequency was observed in recovering subjects $[42,115]$. It seems that the increase in gain was not only because of better quality of retinal image but also due to other factors such as cortical in origin. In addition, no significant correlation was observed between increased contrast sensitivity and other factors such as age of the patient, time elapsed since surgery, type of cataract, or visual acuity before surgery $[42,115]$. Previous studies have also demonstrated significant plasticity recovery in born blind subjects. Individuals who were born blind were able to accurately perform a cross modal matching task after replacement or correction surgery [42]. It appears that these patients in the recovery stage have developed the ability to successfully incorporate visual input data into various multimodal sensory representations. Therefore, it can be assumed that surgical procedures combined with rehabilitative therapies prove to be a promising combination in the restoration of vision for born blind, early blind, and cataract subjects.

Genetic defects in the visual pathway can lead to abnormal wiring and projection of retinal optic nerve fibers to the visual cortex, and they may give rise to some forms of low vision [120]. Genetic abnormality such as albinism is characterized by abnormal synthesis of melanin, and individuals with albinism are associated with decreased visual acuity, hypoplasia, strabismus, and nystagmus, which are known to be the characteristic features of low vision [120]. Normally, optic nerve fibers projecting from the temporal retina to neural circuitry on the ipsilateral side facilitates normal functioning of the visual 
system in humans; however, in albinism, some temporal optic nerve fibers are observed to cross the optic chiasm, projecting temporal hemifield representation superimposed in the nasal hemifield of V1 on the contralateral side [42,120]. This type of circuit arrangement has the ability to distort spatial perception when visual cells in the overlapping region respond to input stimuli from both the hemifields. On the other hand, prior reports have also demonstrated an orderly organization of perceptual visual space in individuals with albinism [121]. It shows the presence of interleaved maps for both temporal and nasal projections in the overlapping area. Previous studies investigating the effect of low vision on neural plasticity in subjects with corneal damage showed no cortical response to visual stimulus for the posterior occipital region, which contains wide representations for foveal vision in normal individuals [122]. It seems that cortical responses are not fully restored post-surgery despite a considerable recovery of visual perception across the entire visual field, and defects in area V1 stem from the absence of small neuronal RFs that are responsible for coding and maintaining information related to high spatial frequency. Prior reports discussing visual cortical responses of blind individuals have exhibited a correlation between (i) visual field loss and decreased visual acuity, and (ii) V1 response amplitude and the extent of V1 responses in individuals with peripheral field loss disorder [42]. It is also evident from prior studies that if the visual cortex is deprived of high resolution input stimulus in the early stages of life, it can alter to accommodate other sensory processing such as auditory stimulus [123]. It is important to note that sight restoring surgery or retinal implants may only be able to achieve partial vision recovery if other non-visual use is irreversible or excludes visual functioning [123]. Previous studies also suggest that the visual circuitry may remain intact and is able to resume normal processing of vision as the lesion projection zone in individuals with central field loss does not respond to visual stimulus, indicating a total absence of remapping of peripheral field into the lesion projection zone [124].

Previous studies investigating the extent of plasticity improvement in subjects with macular degeneration have shown considerable improvement in the reading performance of individuals with central field loss and a strong probability of these patients to benefit from perceptual learning $[58,110]$. Another interesting approach that can benefit individuals with visual field loss who are dependent on visual plasticity is to remap visual stimulus from invisible areas to visible areas [58]. This type of method only becomes effective if the individuals adapt to incorporate displaced and distorted visual input signals with direct stimulus of the visual input $[58,110]$. One drawback in this method is that subjects must learn to differentiate between images that are in the veridical position from remapped images that are in the invisible area, which can sometime lead to confusion. Other methods include the wearing of a head-mounted camera to display visual field images, which is similar to the remapping of an invisible visual field superimposed with the functional central field [42]. It seems that these remapping techniques with the simultaneous training of subjects with visual field loss leads to increased eye scanning movements toward the invisible region, and they have the potential to be the most successful rehabilitative therapy for this defect. Other technologies of retinal remodeling post photoreceptor damage that are currently being explored to treat low vision by restoring, replacing and bypassing damaged retinal fibers to generate input stimulus for visual perception include gene therapy, neuroprotection, optogenetics, stem cell therapy, neural regeneration, and visual prostheses [42,110]. Prosthetics seems to be the most developed of all these approaches, and prior reports have shown the successful ability of planted electrodes to stimulate visual cortical regions of blind subjects $[125,126]$. Sub-retinal and epi-retinal implants along with stimulators are currently being explored in clinical trials as they are less invasive and can significantly enhance the recovery rate of neural processing [125]. One of the major drawbacks of using prostheses is that they exhibit ultra-low values for restored vision that are difficult to measure in clinical trials [125]. Alternative to visual technology, other methods involving auditory sensory and tactile approaches are also being explored for rehabilitative therapy. Previous studies have shown the successful activation of ventral 
visual pathways in systematically trained blind individuals by tactile stimulation methods; however, the cross modal induction of visual areas in blind individuals can trigger more effective processing of spatial elements of auditory inputs that may be playing an important role in the integration of multisensory representation of spatial objects [42,125]. It appears that visual cortical responses to auditory signals in blind individuals is interdependent on accumulated visual experiences that may be similar to visual perception.

It is critical to understand the role of age and visual experience in shaping the neuronal plasticity changes of the visual system. Sight recovery surgery performed for prolonged low vision which emerges early in life, either congenital or late onset, does not have the ability to restore to normal levels of adult vision [120,122,123]. However, partial recovery of visual function in adult stage may be achieved with prolonged deprivation in the early stages of life [123]. Future studies in this aspect may be able to further elucidate our understanding of various factors that determine the conditions that promote or inhibit the rate of recovery in old age and disease. Delayed onset of low vision in aged patients undergoing cataract removal surgery may result in a higher probability of sight restoration [115]. In a similar manner, plastic reorganization in macular degeneration exhibits low levels of plasticity in late onset of low vision; however, oculomotor and functional behavior adaptations are seen even in patients who have low vision in old age $[58,110]$. Long-term alteration of sensory input signals linked with low vision could result in cross modal sharing of visual areas $[42,125]$. It appears that these changes are not directly associated with training; however, certain skills such as reading in subjects with central field loss could be improved by systematic training and perceptual learning. It is most likely that perceptual learning plays an important role in the rehabilitation of patients with low vision, as visual information could be recoded with auditory stimulation. Therefore, we can assume that perceptual learning is essential for sensory stimulations to be successful. One important point to be noted here is that the reorganization of visual circuitry in individuals with low vision may be of great advantage, but this kind of approach may hinder or undermine potential sight recovery therapies at a later stage. On the other hand, reduced neural plasticity and late onset of low vision could render the visual circuitry more receptive to various vision restoration and rehabilitative therapies. Hence, we can assume that the duration of prolonged impairment and age of onset of low vision can either strengthen or undermine the outcome of various therapeutic, surgical, and rehabilitative strategies.

\section{Conclusions}

In this review, I have comprehensively discussed the key differences in the visual circuit mechanism of different species during normal and diseased aging. In order to identify and characterize age-related alterations in various neurodegenerative disorders, it becomes immediately relevant to understand and correlate normal age-related changes in neuroanatomical and behavioral visual circuit mechanisms during healthy aging. This type of approach will further elucidate our understanding of key differences in neural circuit mechanisms between normal and diseased aging as well as highlight the important role of major neural pathways essential for the well-being and healthy aging of the visual system. Although a large volume of data from previous studies have suggested age-induced significant alterations in visual circuit mechanisms that can eventually lead to a variety of neurodegenerative disorders, aged neural circuitry appears to have adapted to compensatory mechanisms as a consequence of accumulated changes over time by reorganizing existing visual pathways and recruiting additional neural networks required to complete a given task. The demand for compensatory neural mechanisms increases with the age and complexity of a given task. Aged neural pathways may have slowed down in their processing speed and may require a longer turnaround time for executing a specific visual task; however, this delayed processing might be necessary to balance the internal parameters of a neural network and may even serve as a means of continuous enhancement of different visual mechanisms within and across species. It is very likely 
that these compensatory mechanisms are differentially processed in higher-order and lower-order visual areas depending on the complexity of the visual function, and at the same time, the compensation mechanism of a visual system may have also played a critical role throughout the evolutionary process of an organism. Therefore, we can assume that aged visual circuitry does not always result in neurological disorders, and the delayed processing due to compensatory mechanisms might actually be essential in overcoming the accumulated changes in the visual system over time. A deeper understanding of how and when age-related changes that occur in V1 and multiple extrastriate visual areas give rise to compensatory processes, and in some cases the insufficiency of these mechanisms resulting in a visual impairment, is of great importance to the design, feasibility, and success of different rehabilitative therapies. Age-dependent changes in neural circuitry can either be due to spontaneous response or visual experience, whereas a visual system that is subjected to diseased aging seems to gradually adapt to alterations introduced by compensatory mechanisms resulting in a significant decline of visual processing and form perception. It appears that age-associated compensatory mechanisms are far more complex than initially understood and may decline in their mechanistic ability with the severity and progression of the disease. However, future studies are necessary to further elucidate underlying key mechanisms that contribute to the transformation of compensatory processes in aged visual circuits to neurological visual impairments.

Funding: This work is supported by the College of Arts and Sciences, American University of Sharjah (AUS). The work in this manuscript is supported, in part, by the Open Access Program from the American University of Sharjah. This manuscript represents the opinions of the author and does not mean to represent the position or opinions of the American University of Sharjah.

Acknowledgments: I dedicate this manuscript to my great loving parents Danka Mohammed Chand Basha and Zabeen Parveen, my loving wife Sana Eunhee Choi, my siblings Ranu (Zaheen Parveen), Sulaik (Danka Mohammed Sulaiman), Chotimaa (Jubee-Veronica) and my family member Ayesha Khan for their unconditional love and support without which this would not have been possible.

Conflicts of Interest: The authors declare no conflict of interest.

\section{References}

1. Betts, L.R.; Taylor, C.P.; Sekuler, A.B.; Bennett, P.J. Aging reduces center-surround antagonism in visual motion processing. Neuron 2005, 45, 361-366. [CrossRef] [PubMed]

2. Betts, L.R.; Sekuler, A.B.; Bennett, P.J. The effects of aging on orientation discrimination. Vis. Res. 2007, 47, 1769-1780. [CrossRef] [PubMed]

3. Del Viva, M.M.; Agostini, R. Visual spatial integration in the elderly. Investig. Ophthalmol. Vis. Sci. 2007, 48, 2940-2946. [CrossRef]

4. Karas, R.; McKendrick, A.M. Age related changes to perceptual surround suppression of moving stimuli. Seeing Perceiving 2012, 25, 409-424. [CrossRef]

5. Brewer, A.A.; Barton, B. Visual cortex in aging and Alzheimer's disease: Changes in visual field maps and population receptive fields. Front. Psychol. 2014, 5, 74. [CrossRef]

6. Schmolesky, M.T.; Wang, Y.; Pu, M.; Leventhal, A.G. Degradation of stimulus selectivity of visual cortical cells in senescent rhesus monkeys. Nat. Neurosci. 2000, 3, 384-390. [CrossRef] [PubMed]

7. Peters, A.; Moss, M.B.; Sethares, C. Effects of aging on myelinated nerve fibers in monkey primary visual cortex. J. Comp. Neurol. 2000, 419, 364-376. [CrossRef]

8. Peters, A.; Moss, M.B.; Sethares, C. The effects of aging on layer 1 of primary visual cortex in the rhesus monkey. Cereb. Cortex. 2001, 11, 93-103. [CrossRef] [PubMed]

9. Yang, Y.; Liang, Z.; Li, G.; Wang, Y.; Zhou, Y. Aging affects response variability of V1 and MT neurons in rhesus monkeys. Brain Res. 2009, 1274, 21-27. [CrossRef]

10. Luebke, J.; Medalla, M.; Amatrudo, J.M.; Weaver, C.M.; Crimins, J.L.; Hunt, B.; Hof, P.R.; Peters, A. Age-related changes to layer 3 pyramidal cells in the rhesus monkey visual cortex. Cereb. Cortex 2015, 25, 1454-1468. [CrossRef]

11. Luebke, J.; Barbas, H.; Peters, A. Effects of normal aging on prefrontal area 46 in the rhesus monkey. Brain Res. Rev. 2010, 62, 212-232. [CrossRef]

12. Luebke, J.I.; Chang, Y.; Moore, T.L.; Rosene, D.L. Normal aging results in decreased synaptic excitation and increased synaptic inhibition of layer 2-3 pyramidal cells in the monkey prefrontal cortex. Neuroscience 2004, 125, 277-288. [CrossRef] [PubMed]

13. Mortazavi, F.; Wang, X.; Rosene, D.L.; Rockland, K.S. White Matter Neurons in Young Adult and Aged Rhesus Monkey. Front. Neuroanat. 2016, 10, 15. [CrossRef] [PubMed] 
14. Hua, T.; Li, X.; He, L.; Zhou, Y.; Wang, Y.; Leventhal, A.G. Functional degradation of visual cortical cells in old cats. Neurobiol. Aging 2006, 27, 155-162. [CrossRef] [PubMed]

15. Hua, T.; Kao, C.; Sun, Q.; Li, X.; Zhou, Y. Decreased proportion of GABA neurons accompanies age-related degradation of neuronal function in cat striate cortex. Brain Res. Bull. 2008, 75, 119-125. [CrossRef] [PubMed]

16. Espinosa, J.S.; Stryker, M. Development and plasticity of the primary visual cortex. Neuron 2012, 75, 230-249. [CrossRef] [PubMed]

17. Polidori, C.; Zeng, Y.C.; Zaccheo, D.; Amenta, F. Age-related changes in the visual cortex: A review. Arch. Gerontol. Geriatr. 1993, 17, 145-164. [CrossRef]

18. Mendelson, J.R.; Wells, E.F. Age-related changes in the visual cortex. Vis. Res. 2002, 42, 695-703. [CrossRef]

19. Miyamoto, S.; Suematsu, N.; Umehira, Y.; Hayashida, Y.; Yagi, T. Age-related changes in the spatiotemporal responses to electrical stimulation in the visual cortex of rats with progressive vision loss. Sci. Rep. 2017, 7, 14165. [CrossRef]

20. Kline, D.W.; Scialfa, C.T. Visual and auditory aging. In Handbook of the Psychology of Aging, 4th ed.; Birren, J.E., Schaie, K.W., Eds.; Academic Press Inc.: San Diego, CA, USA, 1996; pp. 181-203.

21. Weale, R.A. Senile ocular changes, cell death, and vision. In Aging and Human Visual Function; Sekuler, R., Kline, D., Dismukes, K., Eds.; Liss: New York, NY, USA, 1982; pp. 161-171.

22. Weale, R.A. The eye and the aging. Interdisciplinary. Top. Gerontol. 1978, 13, 1-13.

23. Faubert, J.; Overbury, O. Binocular vision in the elderly with adventitious visual impairment: Sometimes one eye is better than two. J. Am. Geriatr. Soc. 2000, 48, 375-380. [CrossRef] [PubMed]

24. Speranza, F.; Moraglia, G.; Schneider, B.A. Age related changes in binocular vision: Detection of noise masked targets in young and old observers. J. Gerontol. Ser. B Psychol. Soc. Sci. 1995, 50, 114-123. [CrossRef] [PubMed]

25. Wagemans, J. Detection of visual symmetries. In Human Symmetry Perception; Tyler, C.W., Ed.; VSP: Utrecht, The Netherlands, 1996; pp. 9-32.

26. Herbert, A.M.; Overbury, O.; Singh, J.; Faubert, J. Aging and bilateral symmetry detection. J Gerontol. B Psychol. Sci. Soc. Sci. 2002, 57, 241-245. [CrossRef] [PubMed]

27. Crassini, B.; Brown, B.; Bowman, K. Age-related changes in contrast sensitivity in central and peripheral retina. Perception 1988, 17, 315-332. [CrossRef]

28. Elliott, D.; Whitaker, D.; MacVeigh, D. Neural contribution to spatiotemporal contrast sensitivity decline in healthy ageing eyes. Vis. Res. 1990, 30, 541-547. [CrossRef]

29. Bennett, P.J.; Sekuler, R.; Sekuler, A.B. The effects of aging on motion detection and direction identification. Vis. Res. 2007, 47, 799-809. [CrossRef]

30. Norman, J.F.; Ross, H.E.; Hawkes, L.M.; Long, J.R. Aging and the perception of speed. Perception 2003, 32, 85-96. [CrossRef]

31. Snowden, R.J.; Kavanagh, E. Motion perception in the ageing visual system: Minimum motion, motion coherence and speed discrimination thresholds. Perception 2006, 35, 9-24. [CrossRef]

32. McIntosh, A.R.; Rajah, M.N.; Lobaugh, N.J. Interactions of prefrontal cortex in relation to awareness in sensory learning. Science 1999, 284, 1531-1533. [CrossRef] [PubMed]

33. Della-Maggiore, V.; Sekuler, A.B.; Grady, C.L.; Bennett, P.J.; Sekuler, R.; McIntosh, A.R. Corticolimbic interactions associated with performance on a short-term memory task are modified by age. J. Neurosci. 2000, 20, 8410-8416. [CrossRef]

34. Bennett, P.J.; Sekuler, A.B.; McIntosh, A.R.; Della Maggiore, V. The effects of aging on visual memory: Evidence for functional reorganization of cortical networks. Acta Psychol. 2001, 107, 249-273. [CrossRef]

35. Faubert, J. Visual perception and aging. Can. J. Exp. Psychol. 2002, 56, 164-176.

36. Wilson, H.R. Cerebral Cortex: Models of Cortical Circuitry; Ulinski, P.S., Jones, E.G., Eds.; Plenum: New York, NY, USA, 1998 ; p. 14.

37. Clifford, C.W.; Vaina, L.M. A computational model of selective deficits in first and second-order motion processing. Vis. Res. 1999, 39, 113-130. [CrossRef]

38. Habak, C.; Faubert, J. Larger effect of aging on the perception of higher-order stimuli. Vis. Res. 2000, 40, 943-950. [CrossRef]

39. Masoro, E.J. Handbook of Physiology in Aging; CRC Press: Boca Raton, FL, USA, 1981.

40. Dakin, S.; Carlin, P.; Hemsley, D. Weak suppression of visual context in chronic schizophrenia. Curr. Biol. 2005, 15, R822-R824. [CrossRef]

41. Markowitz, S.N. Principles of modern low vision rehabilitation. Can. J. Ophthalmol. 2006, 41, 289-312. [CrossRef]

42. Legge, G.E.; Chung, S.T.L. Low Vision and Plasticity: Implications for Rehabilitation. Annu. Rev. Vis. Sci. 2016, 2, 321-343. [CrossRef]

43. Jones, E.G.; Powell, T.P.S. An anatomical study of converging sensory pathways within the cerebral cortex of the monkey. Brain 1970, 93, 793-820. [CrossRef]

44. Dumitriu, D.; Hao, J.; Hara, Y.; Kaufmann, J.; Janssen, W.G.; Lou, W.; Rapp, P.R.; Morrison, J.H. Selective changes in thin spine density and morphology in monkey prefrontal cortex correlate with aging-related cognitive impairment. J. Neurosci. 2010, 30, 7507-7515. [CrossRef]

45. Oga, T.; Aoi, H.; Sasaki, T.; Fujita, I.; Ichinohe, N. Postnatal development of layer III pyramidal cells in the primary visual, inferior temporal, and prefrontal cortices of the marmoset. Front. Neural Circuits 2013, 7, 31-46. [CrossRef]

46. Amatrudo, J.M.; Weaver, C.M.; Crimins, J.L.; Hof, P.R.; Rosene, D.L.; Luebke, J.I. Influence of highly distinctive structural properties on the excitability of pyramidal neurons in monkey visual and prefrontal cortices. J. Neurosci. 2012, 32, 13644-13660. [CrossRef] 
47. Sekuler, R.; Sekuler, A.B. Visual perception and cognition. In Oxford Textbook of Geriatric Medicine; Evans, J.G., Williams, T.F., Beattie, B.L., Michel, J.-P., Wilcock, G.K., Eds.; Oxford University Press: New York, NY, USA, 2000; pp. 874-880.

48. Sekuler, R.; Sekuler, A.B. Age-related changes, optical factors, and neural processes. Encycl. Psychol. 2000, 8, 180-183.

49. Flood, D.G.; Coleman, P.D. Neuron numbers and sizes in aging brain: Comparison of human, monkey, and rodent data. Neurobiol. Aging 1988, 9, 453-464. [CrossRef]

50. Curcio, C.A.; Drucker, D.N. Retinal ganglion cells in Alzheimer's disease and aging. Ann. Neurol. 1993, 33, 248-257. [CrossRef] [PubMed]

51. Curcio, C.A.; Millican, C.L.; Allen, K.A.; Kalina, R.E. Aging of the human photoreceptor mosaic: Evidence for selective vulnerability of rods in central retina. Investig. Ophthalmol. Vis. Sci. 1993, 35, 783-784.

52. Leuba, G. Aging of dendrites in the cerebral cortex of the mouse. Neuropathol. Appl. Neurol. 1983, 9, 467-475. [CrossRef] [PubMed]

53. Severson, J.A. Synaptic regulation of neurotransmitter function in aging. Rev. Biol. Res. Aging 1987, 3, $191-206$.

54. Merigan, W.H.; Katz, L.M.; Maunsell, J.H. The effects of parvocellular lateral geniculate lesions on the acuity and contrast sensitivity of macaque monkeys. J. Neurosci. 1991, 11, 994-1001. [CrossRef]

55. Wang, H.; Xie, X.; Li, X.; Chen, B.; Zhou, Y. Functional degradation of visual cortical cells in aged rats. Brain Res. 2006, 1122, 93-98. [CrossRef]

56. Danka Mohammed, C.P.; Khalil, R. Postnatal Development of Visual Cortical Function in the Mammalian Brain. Front. Syst. Neurosci. 2020, 14, 29. [CrossRef]

57. Yu, B.M.; Afshar, A.; Santhanam, G.; Ryu, S.I.; Shenoy, K.V.; Sahani, M. Extracting dynamical structure embedded in neural activity. In Advances in Neural Information Processing Systems; Weiss, Y., Schölkopf, B., Platt, J., Eds.; MIT Press: Cambridge, MA, USA, 2006; Volume 18, pp. 1545-1552.

58. Yu, D.; Cheung, S.H.; Legge, G.E.; Chung, S.T.L. Reading speed in the peripheral visual field of older adults: Does it benefit from perceptual learning? Vis. Res. 2010, 50, 860-869. [CrossRef] [PubMed]

59. Tanaka, K.; Fukada, Y.; Saito, H.A. Underlying mechanisms of the response specificity of expansion/contraction and rotation cells in the dorsal part of the medial superior temporal area of the macaque monkey. J. Neurophysiol. 1989, 62, 642-656. [CrossRef] [PubMed]

60. Yua, S.; Wanga, Y.; Lia, X.; Zhoua, Y.; Leventhal, A.G. Functional degradation of extrastriate visual cortex in senescent rhesus monkeys. Neuroscience 2006, 140, 1023-1029. [CrossRef] [PubMed]

61. Leventhal, A.G.; Wang, Y.; Pu, M.; Zhou, Y.; Ma, Y. GABA and its agonists improved visual cortical function in senescent monkeys. Science 2003, 300, 812. [CrossRef]

62. Tadin, D.; Blake, R. Motion perception getting better with age. Neuron 2005, 45, 325-332. [CrossRef]

63. Field, D.J.; Hayes, A.; Hess, R.F. Contour integration by the human visual system: Evidence for a local "association field". Vis. Res. 1993, 33, 173-193. [CrossRef]

64. Saarinen, J.; Levi, D.M. Integration of local features into a global shape. Vis. Res. 2001, 41, 1785-1790. [CrossRef]

65. Li, W.; Gilbert, C.D. Global contour saliency and local collinear interactions. J. Neurophysiol. 2002, 88, 2846-2856. [CrossRef]

66. Gilbert, C.D.; Wiesel, T.N. Columnar specificity of intrinsic horizontal and corticocortical connections in cat visual cortex. J. Neurosci. 1989, 9, 2432-2442. [CrossRef]

67. Kapadia, M.K.; Ito, M.; Gilbert, C.D.; Westheimer, G. Improvement in visual sensitivity by changes in local context: Parallel studies in human observers and in V1 of alert monkeys. Neuron 1995, 15, 843-856. [CrossRef]

68. Das, A.; Gilbert, C.D. Receptive field expansion in adult visual cortex is linked to changes in strength of cortical connections. J. Neurophysiol. 1995, 74, 779-792. [CrossRef] [PubMed]

69. Karas, R.; McKendrick, A.M. Aging alters surround modulation of perceived contrast. J. Vis. 2009, 9, 1-9. [CrossRef] [PubMed]

70. Tadin, D.; Lappin, J.S.; Gilroy, L.A.; Blake, R. Perceptual consequences of centre-surround antagonism in visual motion processing. Nature 2003, 424, 312-315. [CrossRef] [PubMed]

71. Zenger-Landolt, B.; Heeger, D.J. Response suppression in v1 agrees with psychophysics of surround masking. J. Neurosci. 2003, 23, 6884-6893. [CrossRef] [PubMed]

72. Bair, M.J.; Robinson, R.L.; Katon, W.; Kroenke, K. Depression and pain comorbidity: A literature review. Arch. Intern. Med. 2003, 163, 2433-2445. [CrossRef]

73. Petrov, Y.; McKee, S.P. The time course of contrast masking reveals two distinct mechanisms of human surround suppression. J. Vis. 2009, 21, 1-11. [CrossRef]

74. Tadin, D.; Kim, J.; Doop, M.L.; Gibson, C.; Lappin, J.S.; Blake, R.; Park, S. Weakened center-surround interactions in visual motion processing in schizophrenia. J. Neurosci. 2006, 26, 11403-11412. [CrossRef]

75. Sekuler, R.; Sekuler, A.B. Visual perception and cognition. In Oxford Textbook of Geriatric Medicine; Evans, J.G., Williams, T.F., Eds.; Oxford University Press: Oxford, UK, 1992; pp. 575-580.

76. Satorre, J.; Cano, J.; Reinoso-Suarez, F. Stability of the neuronal population of the dorsal lateral geniculate nucleus (dLGN) of aged rats. Brain Res. 1985, 339, 375-377. [CrossRef]

77. Celesia, G.G.; Daly, R.F. Effects of aging on visual evoked responses. Arch. Neurol. 1987, 34, 403-407. [CrossRef]

78. Contestabile, M.T.; Suppressa, F.; Tonelli, G.; Giorgi, D.; Antonnicola, G.; D'Alaba, E. The influence of age on the flash visual evoked potentials. Acta Ophthalmol. Scand. 1995, 73, 308-312. [CrossRef] 
79. Pakkenberg, B.; Gundersen, H.J.G. Neocortical neuron number in humans: Effects of sex and age. J. Comp. Neurol. 1997, 384, 312-320. [CrossRef]

80. Salthouse, T.A. Cognitive competence and expertise aging. In Handbook of the Psychology of Aging; Birren, J.E., Schaie, K.W., Eds.; Academic Press: San Diego, CA, USA, 1990; pp. 310-319.

81. Crossland, M.D.; Morland, A.B.; Feely, M.P.; von dem Hagen, E.; Rubin, G.S. The effect of age and fixation instability on retinotopic mapping of primary visual cortex. Investig. Ophthalmol. Vis. Sci. 2008, 49, 3734-3739. [CrossRef] [PubMed]

82. Tang, Y.; Zhou, Y. Age-related decline of contrast sensitivity for second-order stimuli: Earlier onset, but slower progression, than for first-order stimuli. J. Vis. 2009, 9, 18. [CrossRef] [PubMed]

83. Hubel, D.H.; Wiesel, T.N. Receptive fields and functional architecture of monkey striate cortex. J. Physiol. 1968, 195, 215-243. [CrossRef]

84. Dickstein, D.L.; Kabaso, D.; Rocher, A.B.; Luebke, J.I.; Wearne, S.L.; Hof, P.R. Changes in the structural complexity of the aged brain. Aging Cell 2007, 6, 275-284. [CrossRef]

85. Gazzaley, A.H.; Siegel, S.J.; Kordower, J.H.; Mufson, E.J.; Morrison, J.H. Circuit-specific alterations of N-methyl-D-aspartate receptor subunit 1 in the dentate gyrus of aged monkeys. Proc. Natl. Acad. Sci. USA 1996, 93, 3121-3125. [CrossRef]

86. Hof, P.R.; Duan, H.; Page, T.L.; Einstein, M.; Wicinski, B.; He, Y.; Erwin, J.M.; Morrison, J.H. Age-related changes in GluR2 and NMDAR1 glutamate receptor subunit protein immunoreactivity in corticocortically projecting neurons in macaque and patas monkeys. Brain Res. 2002, 928, 175-186. [CrossRef]

87. Siu, C.R.; Beshara, S.P.; Jones, D.G.; Murphy, K.M. Development of glutamatergic proteins in human visual cortex across the lifespan. J. Neurosci. 2017, 37, 6031-6042. [CrossRef]

88. Abuleil, D.; McCulloch, D.L.; Thompson, B. Older Adults Exhibit Greater Visual Cortex Inhibition and Reduced Visual Cortex Plasticity Compared to Younger Adults. Front. Neurosci. 2019, 13, 607. [CrossRef]

89. Wang, Z.; Yu, S.; Fu, Y.; Tzvetanov, T.; Zhou, Y. Aging Potentiates Lateral but Not Local Inhibition of Orientation Processing in Primary Visual Cortex. Front. Aging Neurosci. 2018, 10, 14. [CrossRef] [PubMed]

90. Arani, E.; van Ee, R.; van Wezel, R. Changes in low-level neural properties underlie age-dependent visual decision making. Sci. Rep. 2018, 8, 10789. [CrossRef] [PubMed]

91. Hoshino, O. Ambient gaba responsible for age-related changes in multistable perception. Neural Comput. 2013, 25, 1164-1190. [CrossRef] [PubMed]

92. Serences, J.T.; Yantis, S. Selective visual attention and perceptual coherence. Trends Cogn. Sci. 2006, 10, 38-45. [CrossRef] [PubMed]

93. Nguyen, B.N.; McKendrick, A.M. Visual Contextual Effects of Orientation, Contrast, Flicker, and Luminance: All Are Affected by Normal Aging. Front. Aging Neurosci. 2016, 8, 79. [CrossRef]

94. McKendrick, A.M.; Chan, Y.M.; Nguyen, B.N. Spatial vision in older adults: Perceptual changes and neural bases. Ophthalmic Physiol. Opt. 2018, 38, 363-375. [CrossRef]

95. Samuel, I.B.H.; Wang, C.; Burke, S.E.; Kluger, B.; Ding, M. Compensatory Neural Responses to Cognitive Fatigue in Young and Older Adults. Front. Neural Circuits 2019, 13, 12. [CrossRef]

96. Wang, Z.; Yao, Z.; Yuan, N.; Liang, Z.; Li, G.; Zhou, Y. Declined contrast sensitivity of neurons along the visual pathway in aging cats. Front. Aging Neurosci. 2014, 6, 163. [CrossRef]

97. Gutierrez, A.; Khan, Z.U.; Miralles, C.P.; Mehta, A.K.; Ruano, D.; Araujo, F.; Vitorica, J.; De Blas, A.L. GABAA receptor subunit expression changes in the rat cerebellum and cerebral cortex during aging. Brain Res. Mol. Brain Res. 1997, 45, 59-70. [CrossRef]

98. Ling, L.L.; Hughes, L.F.; Caspary, D.M. Age-related loss of the GABA synthetic enzyme glutamic acid decarboxylase in rat primary auditory cortex. Neuroscience 2005, 132, 1103-1113. [CrossRef]

99. Taylor, J.-P.; Firbank, M.J.; He, J.; Barnett, N.; Pearce, S.; Livingstone, A.; Vuong, Q.C.; McKeith, I.G.; O’Brien, J.T. Visual cortex in dementia with Lewy bodies: Magnetic resonance imaging study. Br. J. Psychiatry 2012, 200, 491-498. [CrossRef]

100. Khundakar, A.A.; Hanson, P.S.; Erskine, D.; Lax, N.Z.; Roscamp, J.; Karyka, E.; Tsefou, E.; Singh, P.; Cockell, S.; Gribben, A.; et al. Analysis of primary visual cortex in dementia with Lewy bodies indicates GABAergic involvement associated with recurrent complex visual hallucinations. Acta Neuropathol. Commun. 2016, 4, 66. [CrossRef] [PubMed]

101. Firbank, M.J.; Parikh, J.; Murphy, N.; Killen, A.; Allan, C.L.; Collerton, D.; Blamire, A.M.; Taylor, J.-P. Reduced occipital GABA in Parkinson disease with visual hallucinations. Neurology 2018, 91, e675-e685. [CrossRef] [PubMed]

102. Reynaud, A.; Tang, Y.; Zhou, Y.; Hess, R.F. Second-order visual sensitivity in the aging population. Aging Clin. Exp. Res. 2018, 31, 705-716. [CrossRef] [PubMed]

103. Brewer, A.A.; Barton, B. Changes in Visual Cortex in Healthy Aging and Dementia; Open Access Peer-Reviewed Chapter 12; IntechOpen Limited: London, UK, 2016. [CrossRef]

104. Krajcovicova, L.; Barton, M.; Elfmarkova-Nemcova, N.; Mikl, M.; Marecek, R.; Rektorova, I. Changes in connectivity of the posterior default network node during visual processing in mild cognitive impairment: Staged decline between normal aging and Alzheimer's disease. J. Neural Transm. 2017, 124, 1607-1619. [CrossRef] [PubMed]

105. Mohammed, C.P.D.; Rhee, H.; Phee, B.; Kim, K.; Kim, H.; Lee, H.; Park, J.H.; Jung, J.H.; Kim, J.Y.; Kim, H.; et al. miR-204 downregulates EphB2 in aging mouse hippocampal neurons. Aging Cell 2016, 15, 380-388. [CrossRef]

106. Danka Mohammed, C.P.; Park, J.S.; Nam, H.G.; Kim, K. MicroRNAs in brain aging. Mech. Ageing Dev. 2017, 168, 3-9. [CrossRef] 
107. Hernández-Zimbrón, L.F.; Gorostieta-Salas, E.; Díaz-Hung, M.L.; Pérez-Garmendia, R.; Gevorkian, G.; Quiroz-Mercado, H. Beta Amyloid Peptides: Extracellular and Intracellular Mechanisms of Clearance in Alzheimer's Disease. In Update on Dementia; Moretti, D., Ed.; IntechOpen Limited: London, UK, 2016. [CrossRef]

108. Coppen, E.M.; Grond, J.V.; Hafkemeijer, A.; Barkey Wolf, J.J.H.; Roos, R.A.C. Structural and functional changes of the visual cortex in early Huntington's disease. Hum. Brain Mapp. 2018, 39, 4776-4786. [CrossRef]

109. Dhalla, A.; Pallikadavath, S.; Hutchinson, C.V. Visual Dysfunction in Huntington's Disease: A Systematic Review. J. Huntingt. Dis. 2019, 8, 233-242. [CrossRef]

110. Chung, S.T.; Levi, D.M.; Tjan, B.S. Learning letter identification in peripheral vision. Vis. Res. 2005, 45, 1399-1412. [CrossRef]

111. Gold, J.; Bennett, P.J.; Sekuler, A.B. Signal but not noise changes with perceptual learning. Nature 1999, 402, 176-178. [CrossRef]

112. Lu, Z.-L.; Dosher, B.A. Perceptual learning retunes the perceptual template in foveal orientation identification. J. Vis. 2004, 4, 44-56. [CrossRef] [PubMed]

113. Andersen, G.J.; Ni, R.; Bower, J.D.; Watanabe, T. Perceptual learning, aging, and improved visual performance in early stages of visual processing. J. Vis. 2010, 10, 4. [CrossRef] [PubMed]

114. Merabet, L.B.; Hamilton, R.; Schlaug, G.; Swisher, J.D.; Kiriakopoulos, E.T.; Pitskel, N.B.; Kauffman, T.; Pascual-Leone, A. Rapid and reversible recruitment of early visual cortex for touch. PLoS ONE 2008, 3, e3046. [CrossRef]

115. Kalia, A.; Lesmes, L.A.; Dorr, M.; Gandhi, T.K.; Chatterjee, G.; Ganesh, S.; Bex, P.J.; Sinha, P. Development of pattern vision following early and extended blindness. Proc. Natl. Acad. Sci. USA 2014, 111, 2035-2039. [CrossRef] [PubMed]

116. Wiesel, T.N.; Hubel, D.H. Binocular interaction in striate cortex of kittens reared with artificial squint. J. Neurophysiol. 1965, 28, 1029-1040. [CrossRef]

117. Wiesel, T.N.; Hubel, D.H. Extent of recovery from the effects of visual deprivation in kittens. J. Neurophysiol. 1965, $28,1060-1072$. [CrossRef]

118. Levi, D.M.; Li, R.W. Perceptual learning as a potential treatment for amblyopia: A mini-review. Vis. Res. 2009, 49, 2535-2549. [CrossRef]

119. Hess, R.F.; Mansouri, B.; Thompson, B. Restoration of binocular vision in amblyopia. Strabismus 2011, 19, 110-118. [CrossRef]

120. Jacobson, S.G.; Cideciyan, A.V.; Roman, A.J.; Sumaroka, A.; Schwartz, S.B.; Heon, E.; Hauswirth, W.W. Improvement and decline in vision with gene therapy in childhood blindness. N. Engl. J. Med. 2015, 372, 1920-1926. [CrossRef]

121. Klemen, J.; Hoffmann, M.B.; Chambers, C.D. Cortical plasticity in the face of congenitally altered input into V1. Cortex 2012, 48, 1362-1365. [CrossRef]

122. Levin, N.; Dumoulin, S.O.; Winawer, J.; Dougherty, R.F.; Wandell, B.A. Cortical maps and white matter tracts following long period of visual deprivation and retinal image restoration. Neuron 2010, 65, 21-31. [CrossRef] [PubMed]

123. Dormal, G.; Lepore, F.; Harissi-Dagher, M.; Albouy, G.; Bertone, A.; Rossion, B.; Collignon, O. Tracking the evolution of crossmodal plasticity and visual functions before and after sight restoration. J. Neurophysiol. 2015, 113, 1727-1742. [CrossRef] [PubMed]

124. Liu, T.; Cheung, S.-H.; Schuchard, R.A.; Glielmi, C.B.; Hu, X.; He, S.; Legge, G.E. Incomplete cortical reorganization in macular degeneration. Investig. Ophthalmol. Vis. Sci. 2010, 51, 6826-6834. [CrossRef] [PubMed]

125. Stingl, K.; Bartz-Schmidt, K.U.; Besch, D.; Chee, C.K.; Cottriall, C.L.; Gekeler, F.; Groppe, M.; Jackson, T.L.; MacLaren, R.E.; Koitschev, A.; et al. Subretinal visual implant Alpha IMS—Clinical trial interim report. Vis. Res. 2015, 111, 149-160. [CrossRef]

126. Plow, E.B.; Obretenova, S.N.; Fregni, F.; Pascual-Leone, A.; Merabet, L.B. Comparison of visual field training for hemianopia with active versus sham transcranial direct cortical stimulation. Neurorehabilit. Neural Repair 2012, 26, 616-626. [CrossRef] 